\title{
SOME THEOREMS ON CLASSES OF RECURSIVELY ENUMERABLE SETS $\left({ }^{1}\right)$
}

\author{
BY \\ J. C. E. DEKKER AND J. MYHILL
}

Introduction. We are concerned with non-negative integers (numbers), collections of numbers (sets) and collections of sets (classes). The intuitive idea of an effectively generated set is made more precise by the notion of a recursively enumerable set. Similarly, one can make the intuitive idea of an effectively generated class of sets more precise by the notion of a recursively enumerable class. This was first done in [2] and [16]. The two main objectives of our paper are:

(1) to extend the results concerning recursively enumerable classes obtained by Rice in [16].

(2) to start a classification of recursively enumerable classes which parallels the classification of recursively enumerable sets initiated by Post in [15].

A mapping of numbers (or ordered $n$-tuples of numbers) onto numbers is called a function. Numbers and functions are denoted by small Latin letters, sets by small Greek letters and classes by capital Latin letters. ' $\alpha-\beta$ ' stands for the set of all numbers which belong to $\alpha$, but not to $\beta$, while ' $A-B$ ' stands for the class of all sets which belong to $A$, but not to $B$. We denote the range of the function $f(x)$ by ' $\rho f(x)$ ' or ' $\rho f$ '. If $\alpha$ is the range of the everywhere defined function $f(x)$, we say that $\alpha$ can be generated by $f(x)$. Let $g(n, x)$ be defined for every ordered pair of numbers and let $A$ be the class of all sets which occur at least once in the sequence $\rho g(0, x), \rho g(1, x), \cdots$. We say that $A$ can be generated by $g(n, x)$.

A set is recursively enumerable (r.e.) if it is empty or it can be generated by a recursive function of one variable. Similarly, a class of r.e. sets is recursively enumerable, if it is empty or consists only of the empty set, or the class of its nonempty members can be generated by a recursive function of two variables. Let ' $F$ ' stand for the class of all r.e. sets and ' $Q$ ' for the class of all finite sets. Then $Q$ is a proper subclass of $F$ and it is easily seen that both $F$ and $Q$ are r.e. classes [2, T2.2].

A set is called decidable or recursive if there exists a recursive procedure which enables us to test membership in the set, i.e., if its characteristic function is recursive. A decidable set is said to have a solvable decision problem, and an undecidable set an unsolvable decision problem. The class of all recursive sets is denoted by ' $E$ '; this class properly includes $Q$ and is properly

Received by the editors, January 19, 1957.

(1) This paper was written while the first-named author was engaged in research supported by National Science Foundation grant NSF G-1978. 
included in $F$. A set in $F-E$ has therefore an unsolvable decision problem in spite of the fact that it can be generated by a recursive function. A class of r.e. sets is called decidable, if there exists a recursive procedure which enables us to determine for any given r.e. set whether it belongs to the class. To make the meaning of 'given' more precise we use a sequence $\omega_{0}, \omega_{1}, \ldots$ in which all r.e. sets are effectively generated. We now consider a r.e. set $\alpha$ as given if we know a number $i$ such that $\alpha=\omega_{i}$. For every subclass $S$ of $F$ we define

$$
\theta S=\left\{n \mid \omega_{n} \in S\right\} \text {. }
$$

The class $S$ of r.e. sets is therefore decidable if and only if the set $\theta S$ is recursive.

From now on the word "class" will only be used in the restricted sense of "class of r.e. sets" and capital Latin letters will always denote subclasses of $F$. The empty class and the class $F$ itself are called the trivial classes. Rice proved in [16] that the only decidable classes are the two trivial ones. In particular there is no recursive procedure for testing whether any given r.e. set is finite; in other words, the set $\theta Q$ is not recursive. In his 1944 paper [15] Post discussed the notion of the degree of unsolvability of an unsolvable decision problem and he raised the question whether the decision problem of $\theta Q$ is of higher degree of unsolvability than that of any set in $F-E$.

§I contains a summary of concepts and results which will be used in the rest of the paper. A short proof of Rice's theorem is given in §II. In §III we obtain lower bounds for the degrees of unsolvability of $\theta S$ for certain choices of the class $S$; we also determine the degree of unsolvability of $\theta Q$. The sets $\alpha$ and $\beta$ are called separable if they can be separated by r.e. sets. The intuitive meaning of " $\alpha$ and $\beta$ are separable" is that when restricted to the union of $\alpha$ and $\beta$, the decision problem of $\alpha$ is solvable. The theorem that every nontrivial class is undecidable can now be phrased as follows: the sets $\theta S$ and $\theta(F-S)$ are not separable for any nontrivial class $S$. In $\S I V$ some conditions are discussed under which the classes $S$ and $T$ are such that $\theta S$ and $\theta T$ are separable. In $\S \mathrm{V}$ a topology is imposed on the class $F$ by specifying certain of its subclasses as open; $F$ turns out to be a connected space when topologized in this manner. Let us call a mapping $\Phi$ from $F$ into itself an effective operation on $F$ if $\Phi\left(\omega_{n}\right)=\omega_{f(n)}$ for some recursive function $f(n)$. It is shown that every effective operation on $F$ is continuous. This fact enables us to give one more proof of the theorem that $F$ has no nontrivial decidable subclasses. The last two sections deal with the classification of classes. A class $A$ is called productive if given a r.e. subclass $S$ of $A$ one can effectively find a set in $A-S$, i.e., a witness to the fact that $S$ is properly included in $A$. Productive classes are considered in $\S \mathrm{VI}$; as examples of such classes we mention $E-Q, F-Q$ and $F-E$. In $\S$ VII r.e. classes are classified in terms of the nature of their complements in $F$.

I. Preliminaries. The sum (i.e., union) of two sets (or classes) is denoted 
by ' + ' and the product (i.e., intersection) of two sets (or classes) by '.' or simply juxtaposition. ' $C$ ' stands for inclusion and ' $C_{+}$' for proper inclusion. In the composition of two or more functions association is to the right unless otherwise indicated. For example, ' $f g h(x, y)$ ' means the same as ' $f(g(h(x, y)))$ '. The abbreviations ' $\rho f^{\prime}$ ', ' $Q$ ', ' $E$ ', ' $F$ ', ' $\alpha-\beta$ ', ' $A-B$ ' were explained in the introduction.

Notations.

$o=$ the empty set,

$\epsilon=$ the set of all numbers,

$\left(a_{0}, a_{1}, \cdots\right)=$ the set consisting of $a_{0}, a_{1}, \cdots$,

$\left[\alpha_{0}, \alpha_{1}, \cdots\right]=$ the class consisting of $\alpha_{0}, \alpha_{1}, \cdots$,

$\alpha^{\prime}=\{x \mid x \notin \alpha\}$,

$A^{\prime}=\left\{\sigma^{\prime} \mid \sigma \in A\right\}$,

$\delta f=$ the domain of $f$,

$j(x, y)=x+(x+y)(x+y+1) / 2$,

$k(n)=(\mu x)(\exists y)[j(x, y)=n]$,

$l(n)=(\mu y)(\exists x)[j(x, y)=n]$.

The functions $j, k$ and $l$ are primitive recursive; $j$ maps the collection of all ordered pairs of numbers one-to-one onto $\epsilon$, while $k$ and $l$ have the property $j(k(n), l(n))=n$.

We recall that both $E$ and $F$ are closed under union and intersection; $E$ is closed under complementation, but $F$ is not; $E C_{+} F$. The most important. relation between $E$ and $F$ is: $\alpha \in E \leftrightarrow \alpha \in F \& \alpha^{\prime} \in F$. It follows that $F-E$ consists of exactly those r.e. sets whose complements are not r.e. This led Post in [15] to classify the sets in $F-E$ in terms of the manner in which their complements deviate from recursive enumerability.

Proposition 1.1. There exist primitive recursive functions $r(x)$ and $m(n, x, y)$ such that the partial recursive function of two variables

$$
q_{n}(x)=r(\mu y)[m(n, x, y)=0]
$$

has the two properties:

(1) for any partial recursive function $f(x)$ there exist infinitely many numbers $n$ such that $f(x)=q_{n}(x)$ and given the recursion equations defining $f(x)$ one can effectively find an infinite r.e. set of such numbers $n$,

(2) $q_{0}(x)$ is nowhere defined, i.e., $\delta q_{0}=o$.

Proof. This is an immediate corollary of a result due to Kleene $[7$, p. 330 , Theorem XIX].

There are obviously denumerably many pairs of primitive recursive functions $r(x)$ and $m(n, x, y)$ with the properties mentioned above. From now on, however, we shall use ' $r(x)$ ' and ' $m(n, x, y)$ ' for one specific pair of such functions and ' $q_{n}(x)$ ' or ' $q(n, x)$ ' only for the partial recursive function $r(\mu y)[m(n, x, y)=0]$. 
Notation. $\omega_{n}=\rho q_{n}$.

It follows from Proposition 1.1 that $\left\{\omega_{n}\right\}$ is a sequence of r.e. sets in which every r.e. set occurs infinitely many times and in which $\omega_{0}$ is the empty set.

Notation. $\kappa=\left\{j(x, y) \mid x \in \omega_{y}\right\}$.

The set $\kappa$ will be referred to as the complete set. It is readily proved [cf. 15 , p. 295] that this set belongs to $F-E$.

DEFINITION. The condition $\Phi(x, y)$ is called recursive (recursively en:ımer$a b l e)$ if the set $\{j(x, y) \mid \Phi(x, y)\}$ is recursive (respectively r.e.). The condition $\Psi(x, y, z)$ is called recursive (recursively enumerable) if the set

$$
\{j(j(x, y), z) \mid \Psi(x, y, z)\}
$$

is recursive (respectively r.e.).

Proposition 1.2. There exists a recursive condition $\Gamma(x, y, z)$ and a r.e. condition $\Delta(x, y)$ such that

$$
x \in \omega_{y} \leftrightarrow(\exists z) \Gamma(x, y, z) \leftrightarrow \Delta(x, y) .
$$

Proof. Let $\kappa$ be the range of the one-to-one recursive function $h(x)$. Abbreviating $j(x, y)=h(z)$ by ' $\Gamma(x, y, z)$ ' and $(\exists z) \Gamma(x, y, z)$ by ' $\Delta(x, y)$ ' we see that $\Gamma$ and $\Delta$ satisfy the requirements.

Let us assume that a one-to-one recursive function $h(x)$ ranging over $\kappa$ is specified once and for all. We agree to use the letters ' $\Gamma$ ' and ' $\Delta$ ' only for the conditions defined in terms of $h(x)$ as in the proof of Proposition 1.2.

Definition. Let $f(x)$ be a partial recursive function, $\alpha$ a r.e. set and $\Phi(x, y)$ a r.e. condition. The number $n$ is called an index of

$f(x)$ if $f(x)=q_{n}(x)$,

$\alpha$ if $\alpha=\omega_{n}$,

$\Phi$ if $\{j(x, y) \mid \Phi(x, y)\}=\omega_{n}$.

Proposition 1.3. Given any (index of a) r.e. condition $\Phi(x, y)$ one can effectively find an infinite r.e. set of numbers $k$ such that

$$
x \in \omega_{k} \leftrightarrow \Phi(x, k) .
$$

Added in proof. The proof given here is incorrect. However, it is easy to give a correct proof using (1) of Proposition 1.1 above, and [13, p. 104, Theorem 15].

Proof. Given an index of the r.e. condition $\Phi$ one can effectively find a number $k$ such that (1) holds, say $k_{0}$ by $[13$, p. 101 , Theorem 8$]$. One can now obtain an index of the r.e. condition $\Phi(x, y) \& y>k_{0}$ and therefore also a number $k$ such that $x \in w_{k} \leftrightarrow \Phi(x, k) \& k>k_{0}$, say $k_{1}$. Continuing this procedure one obtains a strictly increasing recursive function $k_{n}$ such that

$$
t \in \rho k_{n} \rightarrow\left[x \in \omega_{t} \leftrightarrow \Phi(x, t)\right] .
$$

CoRollary. Given any (index of a) recursive function $f(n)$ one can effectively find an infinite r.e. set of numbers $k$ such that $\omega_{k}=\omega_{f(k)}$. 
Proof. Take $\Phi(x, y)=\Delta(x, f(y))$.

Proposition 1.4. There exists a partial recursive function $p(n)$ such that

$$
\delta q_{n} \neq o \rightarrow\left\{\begin{array}{l}
(1) p(n) \text { is defined, } \\
(2) q_{p(n)}(x) \text { is a recursive function, } \\
(3) \rho q_{p(n)}=\rho q_{n} .
\end{array}\right.
$$

Proof. For any partial recursive function $f(x)$ defined for at least one value of $x$ one can effectively find a recursive function with the same range as $f(x)$ by $[4$, p. 136 , Proposition A $]$. The desired result follows by Proposition 1.1.

Definition. The sequence $\left\{\alpha_{n}\right\}$ of r.e. sets is recursively enumerable if $\alpha_{n}=\omega_{f(n)}$ for some recursive function $f(n)$. A weak array is a r.e. sequence of nonempty finite sets; the sets $\alpha_{0}, \alpha_{1}, \cdots$ are the rows of the array. The weak array $\left\{\alpha_{n}\right\}$ is discrete if $\alpha_{m}$ and $\alpha_{n}$ are disjoint for $m \neq n$. A weak array $\left\{\alpha_{n}\right\}$ is an array, if the cardinality of $\alpha_{n}$ is a recursive function of $n$.

It is readily seen that the weak array $\left\{\alpha_{n}\right\}$ is an array if and only if there are recursive functions $a(n, x)$ and $b_{n}$ such that for every $n, a(n, 0), \cdots$, $a\left(n, b_{n}-1\right)$ is the enumeration according to size of $\left\{\alpha_{n}\right\}$. A simple example of a weak array which is not an array is as follows. Let $0 \in \alpha \in F-E$ and $\alpha_{n}=\{x \mid x \in \alpha \& x \leqq n\}$. Then $\left\{\alpha_{n}\right\}$ is a weak array and $y$ belongs to $\alpha$ if and only if $y$ belongs to $\alpha_{y}$. Since $\alpha$ is not recursive, $\left\{\alpha_{n}\right\}$ cannot be an array.

Notation. $O=$ empty class of sets.

Definition I. The class $A$ is recursively enumerable if either $A=O$ or $A=[0]$ or there is a recursive function $f_{n}(x)$ of $n$ and $x$ such that

$$
\alpha \in A-[0] \leftrightarrow(\exists n)\left[\alpha=\rho f_{n}\right] .
$$

Definition II. The class $A$ is recursively enumerable if there is a r.e. set $\alpha$ such that $A=\left\{\omega_{x} \mid x \in \alpha\right\}$.

Proposition 1.5. The two definitions of a r.e. class are equivalent.

Proof. Note that

$$
O=\left\{\omega_{x} \mid x \in o\right\},[o]=\left\{\omega_{x} \mid x \in(0)\right\} .
$$

Thus $O$ and $[0]$ are r.e. according to both definitions. Henceforth we assume that $A-[0]$ is nonempty. Let $A$ be r.e. in the sense of Definition I, say $A-[o]=\left[\rho f_{0}, \rho f_{1}, \cdots\right]$ for some recursive function $f_{n}(x)$. By Proposition 1.1 there is a recursive function $g(n)$ such that $f_{n}(x)=q_{g(n)}(x)$. Hence $A-[0]$ $=\left\{\omega_{x} \mid x \in \rho g\right\}$ and $A$ is r.e. in the sense of Definition II. To establish the converse we assume $A=\left\{\omega_{x} \mid x \in \alpha\right\}$ for some r.e. set $\alpha$. The set

$$
\beta=\left\{n \mid \omega_{n} \neq 0\right\}=\left\{n \mid(\exists x)(\exists y)\left[q_{n}(x)=y\right]\right\}
$$

is clearly r.e. Thus $A-[o]=\left\{\omega_{x} \mid x \in \alpha \beta\right\}$, where $\alpha \beta$ is nonempty and r.e. Let $h(n)$ be a recursive function ranging over $\alpha \beta$ and $p(n)$ a partial recursive 
function with the properties listed in Proposition 1.4. Then

$$
\begin{aligned}
\sigma \in A-[0] & \leftrightarrow(\exists n)\left[\sigma=\omega_{h(n)}\right] \\
& \leftrightarrow(\exists n)\left[\sigma=\rho q_{h(n)}(x)\right] \\
& \leftrightarrow(\exists n)\left[\sigma=\rho q_{p h(n)}(x)\right],
\end{aligned}
$$

where $q_{p h(n)}(x)$ is a recursive function of $n$ and $x$. Thus $A$ is r.e. in the sense of Definition I.

Notations. $P=\left\{\sigma \mid \sigma^{\prime} \in Q\right\}$,

$L(n)=\{\sigma \mid n \in \sigma \in F\}$,

$L(\alpha)=\{\sigma \mid \alpha \subset \sigma \in F\}$,

$K(\alpha)=\{\sigma \mid \sigma \subset \alpha \& \sigma \in F\}$.

Proposition 1.6. The following classes are r.e.

(1) $O, F, E$,

(2) every finite class,

(3) $L(\alpha)$ and $K(\alpha)$ for any $\alpha \in F$.

Proof. (1) $O$ is r.e. by Definition I, $F$ is r.e., since $F=\left\{\omega_{x} \mid x \in \epsilon\right\}$ and $E$ is r.e. by $[2$, p. $82, T 2.2]$.

(2) Left to the reader.

(3) There exist recursive functions $f(n)$ and $g(n)$ such that $\omega_{f(n)}=\alpha+\omega_{n}$ and $\omega_{g(n)}=\alpha \cdot \omega_{n}$. Hence

$$
L(\alpha)=\left\{\omega_{x} \mid x \in \rho f\right\}, \quad K(\alpha)=\left\{\omega_{x} \mid x \in \rho g\right\} .
$$

Proposition 1.7. (1) There exists an array $\left\{\rho_{n}\right\}$ in which all finite sets are enumerated without repetitions.

(2) There exists a recursive function $f_{n}(x)$ which is strictly increasing for every $n$ such that $P$ is enumerated without repetitions in the sequence $\left\{\rho f_{n}\right\}$.

Proof. (1) See $[2$, p. 82, T 2.2] and $[17$, p. 304$]$.

(2) From (1).

Henceforth we shall use ' $\left\{\rho_{n}\right\}$ ' for a specific array which enumerates $Q$ without repetitions such that $\rho_{0}=o$.

Definition. The sequence $\left\{A_{n}\right\}$ of classes is recursively enumerable if there is a recursive function $f(n)$ such that $A_{n}=\left\{\omega_{x} \mid x \in \omega_{f(n)}\right\}$.

It is readily seen that the sum of all classes occurring in a r.e. sequence of r.e. classes is also a r.e. class. Thus the sum of finitely many r.e. classes is again a r.e. class. We shall see in $\S$ VII that the product of two r.e. classes is not necessarily a r.e. class.

Notations. $\theta(\alpha)=\left\{n \mid \omega_{n}=\alpha\right\}$, for $\alpha \in F, \theta A=\left\{n \mid \omega_{n} \in A\right\}$.

Definition. The class $A$ is completely recursively enumerable (c.r.e.) if $\theta A$ is r.e.; $A$ is decidable if $\theta A$ is recursive.

The following statements are immediately verified. $O$ and $F$ are decidable; $A$ is decidable if and only if both $A$ and $F-A$ are c.r.e.; the sum of all classes 
occurring in a r.e. sequence of c.r.e. classes is again c.r.e.; the sum or product of finitely many c.r.e. classes is again a c.r.e. class.

We have used the symbol ' $L(\alpha)$ ' for the class of all r.e. sets which include $\alpha$. We introduce a different notation in the special case that $\alpha$ is finite.

Notation. Let $\alpha \in Q$. Then $T(\alpha)=\{\sigma \mid \alpha \subset \sigma \in F\}$.

Definition. $A$ is called a $\Sigma T$-class if $A=O$ or $A=\sum_{0}^{\infty} T\left(\alpha_{n}\right)$ for some (not necessarily r.e.) sequence $\left\{\alpha_{n}\right\}$ of finite sets.

It follows that $F=T(o)$ is a $\Sigma T$-class and that $\epsilon$ belongs to every nonempty $\Sigma T$-class.

Proposition 1.8. If $\left\{\alpha_{n}\right\}$ is an array, $\sum_{0}^{\infty} T\left(\alpha_{n}\right)$ is a c.r.e. class.

Proof. See Rice [16, pp. 360, 361].

Let $\alpha$ be any set and $S_{\alpha}$ the class of all r.e. sets which are not disjoint from $\alpha$. Then $S_{\alpha}$ is a $\Sigma T$-class and different choices of $\alpha$ yield different classes $S_{\alpha}$. Since $\alpha$ can be chosen in $c$ different ways and $F$ has only $c$ subclasses it follows that there are exactly $c \Sigma T$-classes. Only denumerably many of these have the property that their finite sets can be arranged in an array. For we know that $L(0), L(1), \cdots$ have this property and that there are only denumerably many arrays.

Notations. $\alpha \in \exists$ if $\alpha=\{n|(\exists x)| \Phi(n, x)\}$ for some recursive condition $\Phi(n, x)$,

$\alpha \in \forall$ if $\alpha=\{n \mid(\forall x) \Phi(n, x)\}$ for some recursive condition $\Phi(n, x)$,

$\alpha \in \exists \forall$ if $\alpha=\{n \mid(\exists x)(\forall y) \psi(n, x, y)\}$ for some recursive condition $\psi(n, x, y)$,

$\alpha \in \forall \exists$ if $\alpha=\{n \mid(\forall x)(\exists y) \psi(n, x, y)\}$ for some recursive condition $\psi(n, x, y)$.

We shall refer to the members of $\exists \forall$ and $\forall \exists$ as $\exists \forall$-sets and $\forall \exists$-sets respectively. We recall that $A^{\prime}$ denotes the class of all sets which are complements of sets in $A$. Thus

$$
A^{\prime \prime}=A, \exists=F, \forall=F^{\prime},(\exists \forall)^{\prime}=\forall \exists,(\forall \exists)^{\prime}=\exists \forall .
$$

Proposition 1.9. $F C_{+} \forall \exists \cdot \exists \forall, F^{\prime} C_{+} \forall \exists \cdot \exists \forall$.

Proof. See Mostowski $[10 ; 11]$.

For the notion " $f(x)$ is recursive in $g(x)$ " we refer to [7, p. 295].

Definition. $\alpha$ is (Turing) reducible to $\beta$ (written ' $\alpha \Re \beta$ '), if the characteristic function of $\alpha$ is recursive in the characteristic function of $\beta$.

$\alpha$ is (Turing) equivalent to $\beta$ if $\alpha$ and $\beta$ are reducible to each other.

Post introduced several special types of reducibility in [15]. Of these we need only two.

Definition. $\alpha$ is many-one reducible to $\beta$ (written ' $\alpha Q_{m} \beta$ ') if $x \in \alpha \leftrightarrow f(x) \in \beta$ for some recursive function $f(x)$,

$\alpha$ is one-one reducible to $\beta$ (written ' $\alpha R_{1} \beta$ ') if $x \in \alpha \leftrightarrow f(x) \in \beta$ for some oneto-one recursive function $f(x)$. 
The relation of mutual reducibility is clearly reflexive, symmetric and transitive.

Definition. The degree of $\alpha$ (written 'Deg $\alpha$ ') is the class of all sets which are equivalent to $\alpha$.

It follows that $E$ constitutes one degree; it is called the degree of solvability and denoted by ' $\Delta_{0}$ '. Every other degree consists of nonrecursive sets and is called a degree of unsolvability.

Notation.

$$
\begin{aligned}
& \operatorname{Deg} \alpha \leqq \operatorname{Deg} \beta \text { if } \alpha \Re \beta, \\
& \operatorname{Deg} \alpha<\operatorname{Deg} \beta \text { if } \alpha \Re \beta, \text { but not } \beta R \alpha .
\end{aligned}
$$

Definition. Deg $\alpha$ and Deg $\beta$ are incomparable if neither $\alpha R \beta$ nor $\beta R \alpha$.

The structure of the collection of all degrees was studied by Kleene and Post in [8].

Every set is equivalent to its complement. A degree which can be represented by a set in $F-E$ can therefore also be represented by a set in $F^{\prime}-E$ and conversely. Similarly a degree which contains an $\boldsymbol{\exists} \forall$-set also contains an $\forall \exists$-set and conversely.

Proposition 1.10. (1) Among the degrees represented by sets in $F+F^{\prime}$ there is a highest one (written ' $\Delta_{1}$ ') and this highest degree can be represented by $k$.

(2) Among the degrees represented by sets in $\exists \forall+\forall \exists$ there is a highest one (written ' $\Delta_{2}$ ') and this highest degree is greater than $\Delta_{1}$.

Proof. For (1) see [15, p. 297] and for (2) see [7, p. 315].

We have $\Delta_{0}<\Delta_{1}<\Delta_{2}$. The problem whether more than one degree can be represented by sets in $F-E$ was called Post's problem. It was solved independently by Friedberg [6] and Muchnik [12]. They proved that there is an infinite sequence of r.e. sets with mutually incomparable degrees. Consequently there are denumerably many degrees between $\Delta_{0}$ and $\Delta_{1}$ which can be represented by sets in $F-E$.

Definition. The set $\alpha$ is productive relative to the partial recursive function $p(n)$ if

$$
\omega_{n} \subset \alpha \rightarrow\left\{\begin{array}{l}
(1) p(n) \text { is defined } \\
(2) p(n) \in \alpha-\omega_{n} .
\end{array}\right.
$$

Every partial recursive function $p(n)$ related to $\alpha$ by (1) is a productive function of $\alpha$. A set is productive if it has at least one productive function. A r.e. set with a productive complement is called creative.

It can be shown [cf. 15, p. 295] that the complete set $\kappa$ is creative. Hence $\kappa^{\prime}$ is an example of a productive set with a r.e. complement.

PROPOSITION 1.11. Every productive set has a recursive productive function.

Proof. Let $p(n)$ be one of the productive functions of the productive set $\alpha$. There exists a recursive function $f(n)$ such that 


$$
\omega_{f(n)}=\left\{x \mid n \in \delta p \&\left(x=p(n) \vee x \in \omega_{n}\right)\right\} .
$$

It follows that

(1) $n \in \delta p \rightarrow \omega_{f(n)}=\omega_{n}+(p(n))$,

(2) $n \notin \delta p \rightarrow \omega_{f(n)}=o$,

(3) $n \in \delta p \rightarrow p f(n)$ is defined.

Let

$$
\begin{aligned}
p(x) & =q_{a}(x)=r(\mu y)[m(a, x, y)=0], \\
p f(x) & =q_{b}(x)=r(\mu y)[m(b, x, y)=0], \\
g(x) & =r(\mu y)[m(a, x, y)=0 \vee m(b, x, y)=0] .
\end{aligned}
$$

Since $g(x)$ is a recursive function which for every $x$ equals either $p(x)$ or $p f(x)$, it suffices to show that $p f(x)$ is a productive function of $\alpha$. By (1) $\omega_{x} \subset \alpha \rightarrow \omega_{f(x)} \subset \alpha \rightarrow p f(x) \in \alpha-\left(\omega_{x}+(p(x))\right) \rightarrow p f(x) \in \alpha-\omega_{x}$.

Proposition 1.12. (1) $\alpha$ productive \& $\alpha R_{m} \beta \rightarrow \beta$ productive,

(2) $\alpha$ productive $\leftrightarrow \kappa^{\prime} R_{m} \alpha$,

(3) $\alpha$ productive $\rightarrow \Delta_{1} \leqq$ Deg $\alpha$.

Proof. (1) See [4, p. 140, T 2.8].

(2) In view of (1) and the fact that $\kappa^{\prime}$ is productive, it suffices to establish the conditional from the left to the right. According to [13, Theorem 10] every r.e. set $\alpha$ is many-one reducible to every creative set $\beta$. While the proof of this theorem in [13] uses the fact that $\beta^{\prime}$ is productive and has a recursive productive function, it does not use the recursive enumerability of $\beta\left({ }^{2}\right)$. We infer by Proposition 1.11 that every r.e. set is many-one reducible to every' set with a productive complement. Thus, for any productive set $\gamma$ we have $\kappa R_{m} \gamma^{\prime}$ and $\kappa^{\prime} R_{m} \gamma$.

(3) Immediate from (2).

The converse of (3) is false; for every productive set has an infinite r.e. subset and it can be shown that every degree greater than $\Delta_{0}$ contains an infinite set without any infinite r.e. subset [5, Chapter IX]. On the other hand, every degree $\geqq \Delta_{1}$ can be represented by a productive set according to an unpublished result due to Mrs. C. Karp.

Definition. The sets $\alpha$ and $\beta$ are isomorphic (written ' $\alpha \cong \beta$ ') if there exists a recursive permutation of $\epsilon$ which maps $\alpha$ onto $\beta$. The class $A$ is an isomorphism type if $A$ consists of all sets which are isomorphic with some set $\alpha$.

Every set which is isomorphic with a productive set is again productive and similarly for creative sets.

Proposition 1.13. All creative sets are isomorphic.

Proof. See [13, p. 108, Theorem 19].

The class of all creative sets forms therefore an isomorphism type.

(2) This was pointed out to the authors by S. Tennenbaum. 
II. Decidable classes. As our starting point we choose the fact that there are no decidable classes besides $O$ and $F$. This result can be generalized in different directions (e.g. to Corollary 1 of Theorem 3.1 or to Theorem 4.1), but we first present a proof which is shorter than Rice's original proof. We shall use only two basic theorems of recursion theory, namely the fact that some r.e. sets are not recursive and the existence of a partial recursive function $q(n, x)$ with the properties stated in Proposition 1.1.

THEOREM 2.1. The only decidable classes are $O$ and $F$.

Proof. $O$ and $F$ are decidable, because $\theta O=o$ and $\theta F=\epsilon$. Assume $S \neq O$ and $S \neq F$; let $A$ denote that one of the two nonempty classes $S$ and $F-S$ which contains $o$. Suppose the set $\beta$ belongs to $F-E$ and is the range of the recursive function $b(x)$. There is a nonempty set in $F-A$, say $\gamma$; let $c(x)$ be a recursive function ranging over $\gamma$. Consider the partial recursive function

$$
d_{n}(x)=d(n, x)=c(x) \cdot \operatorname{sg}[(\mu y)\{b(y)=n\}+1] .
$$

By Proposition 1.1 there is a recursive function $f(n)$ such that $d(n, x)$ $=q(f(n), x)$. Hence

$$
\begin{aligned}
& n \in \beta \rightarrow \delta d_{n}=\epsilon \rightarrow \rho d_{n}=\omega_{f(n)}=\gamma \in F-A, \\
& n \in \beta \rightarrow \delta d_{n}=o \rightarrow \rho d_{n}=\omega_{f(n)}=o \in A, \\
& n \in \beta \leftrightarrow f(n) \in \theta(F-A) .
\end{aligned}
$$

If $S$ were decidable the classes $F-S, A$ and $F-A$ would also be decidable; the set $\beta$ would therefore be recursive by (1), contrary to the assumption $\beta \in F-E$. Thus $S$ is undecidable.

III. Theta sets. The set $\sigma$ is called a theta set if $\sigma=\theta S$ for some class $S$; $\sigma$ is a trivial theta set if it equals $o$ or $\epsilon$. By Theorem 2.1 every nontrivial theta set has a degree which exceeds $\Delta_{0}$. The main purpose of this section is to strengthen this result by obtaining higher lower bounds for the degrees of some nontrivial theta sets. A class $S$ is called ancestral if $\sigma \in S \& \sigma \subset \tau \rightarrow \tau \in S$.

Let us consider the following four categories of classes:

(a) $\Sigma T$-classes whose finite sets form an array,

(b) $\Sigma T$-classes whose finite sets do not form an array,

(c) classes which are not ancestral,

(d) classes which contain at least one infinite set without containing any of its finite subsets.

It is clear that every class belongs to at least one of these categories and that every class which is not a $\Sigma T$-class falls under (c) or (d) (possibly both).

TheOREM 3.1. If the class $A$ is not a $\Sigma T$-class, the set $\theta A$ is productive.

Proof. Let $A$ satisfy the hypothesis. By Proposition 1.12 it suffices to prove that $\kappa^{\prime} \mathfrak{R}_{m} \theta A$.

CAse 1. $A$ is not ancestral. Let $\alpha \subset \beta, \alpha \in A, \beta \in F-A$. By Proposition 1.1 


$$
\omega_{f(n)}=\{x \mid x \in \beta \&(n \in \kappa \bigvee x \in \alpha)\}
$$

for some recursive function $f(n)$. Thus,

$$
\begin{aligned}
& n \in \kappa \rightarrow \omega_{f(n)}=\beta \rightarrow f(n) \notin \theta A, \\
& n \in \kappa^{\prime} \rightarrow \omega_{f(n)}=\alpha \rightarrow f(n) \in \theta A, \\
& n \in \kappa^{\prime} \leftrightarrow f(n) \in \theta A, \text { i.e., } \kappa^{\prime} \Re_{m} \theta A .
\end{aligned}
$$

Case 2. $A$ contains an infinite r.e. set without containing any of its finite subsets, say $\sigma$. Let $h(n)$ be a one-to-one recursive function ranging over $\kappa$. For every number $n$, the set

$$
\tau_{n}=\{x \mid x \in \sigma \&(\forall y \leqq x)[h(y) \neq n]\}
$$

is a r.e. subset of $\sigma$; let $g(n)$ be a recursive function such that $\tau_{n}=\omega_{g(n)}$. If $n \in \kappa^{\prime}$ the number $h(y)$ is different from $n$ for all $y$; this implies

$$
n \in \kappa^{\prime} \rightarrow \omega_{\theta(n)}=\sigma \in A .
$$

Now suppose $n \in \kappa$. Then there is a unique $y$ such that $h(y)=n$, say $u_{n}$; thus $x \notin \omega_{g(n)}$ for $x \geqq u_{n}$ and $\omega_{g(n)}$ is a finite subset of $\sigma$. Hence

$$
n \in \kappa \rightarrow \omega_{g(n)} \notin A \text {. }
$$

Combining (1) and (2) we obtain $\kappa^{\prime} \mathfrak{R}_{m} \theta A$.

Corollary 1. For every nontrivial class $A$,

(1) at least one of the two sets $\theta A$ and $\theta(F-A)$ is productive,

(2) A c.r.e. $\rightarrow \theta A$ creative,

(3) $\Delta_{1} \leqq \operatorname{Deg} \theta A$.

Proof. Let $A \neq O, A \neq F$. Every nonempty $\Sigma T$-class contains $\epsilon$. Thus at least one of the two nonempty classes $A$ and $F-A$ is not a $\Sigma T$-class; this implies (1). Suppose $A$ is c.r.e.; then $\theta A$ is r.e., hence not productive. Then $\theta(F-A)$ is productive by (1) and $\theta A$ is creative. This establishes (2). Note that $\theta A$ and $\theta(F-A)$ have the same degree, because they are complementary. Thus (1) implies (3) in view of Proposition 1.12 (3).

Corollary $2\left(^{3}\right)$. A class is c.r.e. if and only if it is a $\Sigma T$-class whose finite sets form an array.

Proof. We only have to prove the "only if" part in view of Proposition 1.8. Let $A$ be c.r.e., i.e., let $\theta A$ be r.e. Then $A$ is a $\Sigma T$-class, otherwise $\theta A$ would be productive. We now use the enumeration $\left\{\rho_{n}\right\}$ of $Q$ mentioned in Proposition 1.7; there exists a recursive function $h(n)$ such that $\rho_{n}=\omega_{h(n)}$. Note that $\rho_{n} \in A \leftrightarrow h(n) \in \theta A$. Using a recursive function ranging over $\theta A$ one can therefore find a recursive function $(u) n$ such that $A \cdot Q=\left[\rho_{u(0)}, \rho_{u(1)}, \cdots\right]$.

(3) R. McNaughton and N. Shapiro obtained this result independently of each other and of the authors. 
Corollari 3. $\alpha$ r.e. $\rightarrow \theta(\alpha)$ productive.

Proof. Let $A=[\alpha]$. If $\alpha$ is finite $A$ is not ancestral, and if $\alpha$ is infinite $A$ contains $\alpha$, but no finite subset of $\alpha$. Anyhow, $A$ is not a $\Sigma T$-class and the set $\theta A=\theta(\alpha)$ is productive.

lote that Theorem 2.1 was not used in the proof of Theorem 3.1 or its first corollary. We claim that Theorem 2.1 is implied by Corollary 1 . Suppose, namely, that there existed a nontrivial decidable class, say $S$. Then $S$ and $F-S$ would be c.r.e. classes, $\theta S$ and $\theta(F-S)$ recursive sets and $\operatorname{Deg} \theta S$ $=\Delta_{0}<\Delta_{1}$. Using any of the three parts of Corollary 1 we obtain a contradiction. We conclude that every nontrivial class is undecidable.

We have seen that $\Delta_{1} \leqq \operatorname{Deg} \theta S$ for every nontrivial class $S$. This raises the question whether the $\leqq$ sign can be strengthened to $=$ or $<$. We shall see that neither is the case as long as all we know about $S$ is that it is nontrivial. $\left({ }^{4}\right)$

Theorem 3.2. $\alpha \in Q \rightarrow \mathrm{D}$ eg $\theta(\alpha)=\Delta_{1}$.

Proof. First assume $\alpha=o$; let $S$ denote the class of all nonempty r.c. sets. Then $S$ is c.r.e., $\theta S$ creative and $\operatorname{Deg} \theta(o)=\operatorname{Deg} \theta S=\Delta_{1}$. Now assume $\alpha \neq 0$, say $\alpha=\left(a_{1}, \cdots, a_{s}\right)$. Then

$$
\theta(\alpha)=\beta \cdot \gamma, \text { where }\left\{\begin{array}{l}
\beta=\left\{x \mid a_{1} \in \omega_{x} \& \cdots \& a_{s} \in \omega_{x}\right\}, \\
\gamma=\left\{x \mid(\forall y)\left(y \in \omega_{x}^{\prime} \vee y=a_{1} \vee \cdots \vee y=a_{s}\right)\right\} .
\end{array}\right.
$$

It is not difficult to verify that $\beta \in \exists$ and $\gamma \in \forall$; thus $\operatorname{Deg} \theta(\alpha) \leqq \Delta_{1}$. By part (3) of Corollary 1 of Theorem 3.1, $\Delta_{1} \leqq \operatorname{Deg} \theta(\alpha)$; hence $\operatorname{Deg} \theta(\alpha)=\Delta_{1}$.

We proceed to determine the degree of $\theta Q$.

Theorem 3.3. $\theta(F-Q)$ is an $\forall \exists$-set to which every $\forall \exists$-set is one-one reducible. Similarly, $\theta Q$ is an $\exists \forall$-set to which every $\exists \forall$-set is one-one reducible.

Proof. The complement of an $\forall \exists$-set is an $\boldsymbol{\exists} \forall$-set and conversely. Moreover, $\alpha R_{1} \beta$ if and only if $\alpha^{\prime} R_{1} \beta^{\prime}$. Finally, $\theta Q$ and $\theta(F-Q)$ are complementary. Thus, the two parts of the theorem are equivalent and we may restrict our attention to the first part.

$$
n \in \theta(F-Q) \leftrightarrow \omega_{n} \notin Q \leftrightarrow(\forall x)(\exists y)\left[y>x \& y \in \omega_{n}\right] .
$$

By Proposition 1.2 there is a recursive condition $\Gamma(y, n, z)$ such that $y \in \omega_{n}$ $\leftrightarrow(\exists z) \Gamma(y, n, z)$. Hence

(4) Let for any family $Q$ of recursive functions of one variable $\theta Q$ denote the set of all numbers $n$ for which $q_{n}(x) \in Q$. Shapiro [18] studies $\theta Q$ for several choices of $Q$. Some of his theorems can be used to obtain information about $\theta S$ for certain choices of $S$. Much of his terminology concerns families of partial recursive functions and is not relevant to classes of r.e. sets. To keep the present paper reasonably self-contained we have not drawn upon his results. 


$$
\begin{aligned}
n \in \theta(F-Q) & \leftrightarrow(\forall x)(\exists y)(\exists z)[y>x \& \Gamma(y, n, z)], \\
& \leftrightarrow(\forall x)(\exists t)[k(t)>x \& \Gamma(k(t), n, l(t))], \\
& \leftrightarrow(\forall x)(\exists t) \Psi(n, x, t)
\end{aligned}
$$

for some recursive condition $\Psi$. Consequently $\theta(F-Q)$ is an $\forall \exists$-set. Now suppose $\alpha$ is any $\forall \exists$-set, say $n \in \alpha \leftrightarrow(\forall x)(\exists y) \Phi(n, x, y)$ for some recursive condition $\Phi$. Then

$$
n \in \alpha \leftrightarrow \text { for infinitely many } x,(\forall z \leqq x)(\exists y) \Phi(n, z, y) .
$$

For $n=0$ we can effectively find a number $f(0)$ such that

$$
\omega_{f(0)}=\{x \mid(\forall z \leqq x)(\exists y) \Phi(0, z, y)\}
$$

and for $n=k+1$ we can effectively find a number $f(k+1)$ such that

$$
f(k)<f(k+1) \& \omega_{f(k+1)}=\{x \mid(\forall z \leqq x)(\exists y) \Phi(k+1, z, y)\} .
$$

It follows that there is a one-to-one recursive function $f(n)$ with the property

$$
\omega_{f(n)}=\{x \mid(\forall z \leqq x)(\exists y) \Phi(n, z, y)\} .
$$

Combining (1) and (2) we obtain

$$
n \in \alpha \leftrightarrow \omega_{f(n)} \in F-Q \leftrightarrow f(n) \in \theta(F-Q) .
$$

Hence $\alpha \Re_{1} \theta(F-Q)$.

Corollary. Deg $\theta Q=\operatorname{Deg} \theta(F-Q)=\Delta_{2}$.

According to the following theorem there are many classes $S$ for which $\Delta_{2} \leqq \operatorname{Deg} \theta S$.

THEOREM 3.4. If the nontrivial class $S$ has the properties

$$
\begin{aligned}
& \sigma \in S \& \beta \in Q \rightarrow \sigma+\beta \in S, \\
& \epsilon \in S,
\end{aligned}
$$

then $\theta Q R_{1} \theta S$ and $\theta(F-Q) R_{1} \theta(F-S)$.

Proof. Let $S$ satisfy the hypotheses. We shall show that every $\forall \exists$-set is one-one reducible to $\theta(F-S)$. Let $\alpha$ be any $\forall \exists$-set, say

$$
n \in \alpha \leftrightarrow(\forall x)(\exists y) \Phi(n, x, y)
$$

for some recursive condition $\Phi$ and let $\tau$ be any specific set in $S$. Then let $f$ be a recursive function such that $\omega_{f(n)}=\{x \mid(\forall z \leqq x)(\exists y) \Phi(n, z, y)\}$.

$$
\begin{aligned}
n \in \alpha & \rightarrow(\forall x)(\forall z \leqq x)(\exists y) \Phi(n, z, y) \\
& \rightarrow\{x \mid(\forall z \leqq x)(\exists y) \Phi(n, z, y)\}=\epsilon \\
& \rightarrow \omega_{f(n)}=\epsilon \\
& \rightarrow \omega_{f(n)}+\tau=\epsilon \in S .
\end{aligned}
$$


There is a recursive function $g(n)$ such that

$$
\begin{aligned}
\omega_{g(0)} & =\omega_{f(0)}+\tau, \\
\omega_{g(n+1)} & =\omega_{f(n+1)}+\tau \& g(n)<g(n+1) .
\end{aligned}
$$

Thus,

$$
n \in \alpha \rightarrow \omega_{o(n)} \notin S \rightarrow g(n) \in \theta(F-S),
$$

where $g(n)$ is a one-to-one recursive function. On the other hand

$$
\begin{aligned}
n \notin \alpha & \rightarrow \text { for only finitely many } x,\left(\forall_{z} \leqq x\right)(\exists y) \Phi(n, z, y) \\
& \rightarrow\left\{x \mid\left(\forall_{z} \leqq x\right)(\exists y) \Phi(n, z, y)\right\} \in Q \\
& \rightarrow \omega_{f(n)} \in Q \\
& \rightarrow \omega_{g(n)}=\omega_{f(n)}+\tau \in S \\
& \rightarrow g(n) \notin \theta(F-S) .
\end{aligned}
$$

Thus, $n \in \alpha \leftrightarrow g(n) \in \theta(F-S)$ and $\alpha R_{1} \theta(F-S)$.

Since the theta sets so far discussed have been either r.e. or productive, the question arises whether this is the case for all theta sets. In view of the characterization of c.r.e. classes in terms of arrays and Theorem 3.1 the only theta sets still to be examined are of the form $\theta S$ where $S$ is a $\Sigma T$-class whose finite sets do not form an array.

THEOREM 3.5. There exists a $\Sigma T$-class $A$ such that $\theta A$ is neither r.e. nor productive.

Proof. By [6] or [12] there is a set in $F-E$ whose degree is less than $\Delta_{1}$, say $\mu$. Let $A$ denote the class of all r.e. sets which contain at least one element of $\mu^{\prime}$. Suppose $A$ were c.r.e.; then the finite sets in $A$ would form an array, say $\left\{\rho_{f(n)}\right\}$, where $f(n)$ is a one-to-one recursive function. Let $g$ be a recursive function such that $(x)=\rho_{g(x)}$. We now have

$$
x \in \mu^{\prime} \leftrightarrow(x) \in A \leftrightarrow(\exists n)[g(x)=f(n)] .
$$

Thus $\mu^{\prime}$ would be r.e. and $\mu$ recursive, contrary to the assumption $\mu \in F-E$. Hence $A$ is not c.r.e. and $\theta A$ not r.e. It remains to be shown that $\theta A$ is not productive. We say that the set $\sigma$ is recursively enumerable in the set $\tau$ if $\sigma$ is the range of some function $h(n)$ which is partial recursive in the characteristic function of $\tau$. We claim

(1) $\alpha \in F \& \alpha^{\prime}$ r.e. in $\beta \rightarrow \alpha \Re \beta$,

(2) $\alpha R_{m} \beta \& \beta$ r.e. in $\gamma \rightarrow \alpha$ r.e. in $\gamma$,

(3) $\theta A$ is r.e. in $\mu$.

The first two statements are easily verified by the reader. Note that

$$
x \in \theta A \leftrightarrow(\exists y)\left[y \in \omega_{x} \cdot \mu^{\prime}\right] \leftrightarrow(\exists n)\left[l(n) \in \omega_{k(n)} \cdot \mu^{\prime} \& x=k(n)\right] .
$$

Let $t(n)$ be the function which equals $k(n)$ if $l(n) \in \omega_{k(n)} \cdot \mu^{\prime}$, but is undefined 
otherwise. Then $\theta A=\rho t$, where $t(n)$ is partial recursive in the characteristic function of $\mu$. This establishes (3). Now suppose $\theta A$ were productive. Then $\kappa^{\prime} R_{m} \theta A$ by Proposition 1.12, and $\kappa^{\prime}$ r.e. in $\mu$ by (2) and (3). Thus $\kappa R \mu$ by (1) contrary to the assumption Deg $\mu<\Delta_{1}$. We conclude that $\theta A$ is not productive.

The results of this section can be summarized in the following diagram. A single-headed arrow from $\alpha$ to $\beta$ indicates that $\alpha$ is reducible to $\beta$, a doubleheaded arrow from $\alpha$ to $\beta$ means that $\alpha$ is reducible to $\beta$, but not conversely, and an arrow with heads at both ends stands for mutual reducibility. $S$ is any class which satisfies the hypotheses of Theorem 3.4.

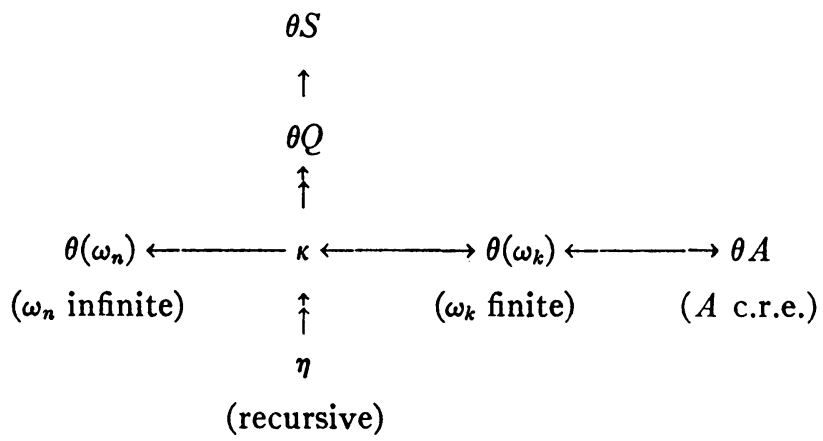

IV. Separable classes. The set $\alpha$ is separable from the set $\beta$ (written ' $\alpha \mid \beta$ ') if there is a partial recursive function $p(x)$ such that

(1) $\alpha+\beta \subset \delta p \& \delta p \subset(0,1)$,

(2) $x \in \alpha+\beta \rightarrow(x \in \alpha \leftrightarrow p(x)=1)$.

If $\alpha \mid \beta$ is false we say that $\alpha$ is inseparable from $\beta$. We can speak of two sets as being separable or inseparable, because the / relation is obviously symmetric. It is readily seen that $\alpha \mid \beta$ is true if and only if there are disjoint r.e. sets $\alpha_{1}$ and $\beta_{1}$ such that $\alpha \subset \alpha_{1}$ and $\beta \subset \beta_{1}$. Any two separable sets are disjoint, but not conversely. For let

$$
\omega_{t} \in F-E, \alpha=\left\{j(x, t) \mid x \in \omega_{t}\right\}, \beta=\left\{j(x, t) \mid x \in \omega_{t}\right\},
$$

then $\rho j(x, t)=\alpha+\beta$ and $x \in \omega_{t} \leftrightarrow j(x, t) \in \alpha$. If $\alpha \mid \beta$ were true, $\omega_{t}$ would be recursive. Thus $\alpha$ and $\beta$ are disjoint, but inseparable. Note that in this example $(\alpha+\beta)^{\prime}$ is infinite.

Definition. The classes $A$ and $B$ are separable (written ' $A \mid B$ ') if $\theta A \mid \theta B$, otherwise inseparable.

As in the case of sets any two separable classes are disjoint, but not conversely. Suppose namely that $i(n)$ is a recursive function such that $\omega_{i(n)}=(n)$ and that $\sigma \in F-E$. Put

$$
A_{\sigma}=\{(n) \mid n \in \sigma\}, \quad B_{\sigma}=\{(n) \mid n \notin \sigma\} .
$$


In view of the fact that

$$
n \in \sigma \leftrightarrow i(n) \in \theta A_{\sigma}, \quad n \in \sigma \leftrightarrow i(n) \in \theta B_{\sigma}
$$

we see that $A_{\sigma} \mid B_{\sigma}$ implies $\sigma \in E$. Thus, $A_{\sigma}$ and $B_{\sigma}$ are disjoint, but inseparable.

A set is recursive if and only if it is separable from its complement. The class $A$ is therefore decidable if and only if $A \mid F-A$. This permits us to rephrase Theorem 2.1 as follows: every nontrivial class is inseparable from its complement in $F$. This leads to the question whether there exists any pair of separable nontrivial classes. We shall see that there exist $c$ such pairs. In this section we wish to study conditions under which two classes are separable.

TheOREM 4.1 $\left(^{5}\right)$. A necessary condition for $A \mid B$ is that no member of one of the two classes $A$ and $B$ be included in any member of the other class.

Proof. Let $p(x)$ be a partial recursive function such that

$$
\theta A+\theta B \subset \delta p, \quad x \in \theta A \leftrightarrow p(x)=1 \& x \in \theta B \leftrightarrow p(x)=0 .
$$

In view of the symmetry of the $\mid$ relation we only prove that no member of $A$ is included in any member of $B$. Suppose $\alpha \in A, \beta \in B$ and $\alpha \subset \beta$. As in the proof of Theorem 3.1 we use a recursive function $f(n)$ with the property

$$
\omega_{f(n)}=\{x \mid x \in \beta \&(n \in \kappa \bigvee x \in \alpha)\} \text {. }
$$

We now have

$$
\begin{aligned}
& n \in \kappa \rightarrow \omega_{f(n)}=\beta \in B, n \notin \kappa \rightarrow \omega_{f(n)}=\alpha \in A, \\
& \rho f \subset \theta A+\theta B \subset \delta p, \\
& n \in \kappa \leftrightarrow f(n) \in \theta B \leftrightarrow p f(n)=0 .
\end{aligned}
$$

Since $p f(n)=0$ is a recursive condition it would follow that $\kappa$ is a recursive set. The assumption $\alpha \in A, \beta \in B, \alpha \subset \beta$ must therefore be false.

Let us examine some of the implications of the necessary condition just obtained. Suppose $A \mid B$. First of all,

$$
\alpha \in A \& \beta \in B \rightarrow \alpha \beta, \quad \alpha+\beta \notin A+B,
$$

since $\alpha \beta$ is included in both $\alpha$ and $\beta$ and $\alpha+\beta$ includes both $\alpha$ and $\beta$. Moreover,

$$
\alpha \in A \rightarrow 0, \epsilon \in B \text {. }
$$

Thus, if $A$ and $B$ are nontrivial and separable, reither $A$ nor $B$ contains o or $\epsilon$. It follows that Theorem 4.1 generalizes Theorem 2.1. For suppose $S$ and $T$ are nontrivial classes, then

(5) Theorems 4.1,4.2 and 4.3 are due to R. McNaughton. Up till now only their statements have been published [9]. We are indebted to McNaughton for his permission to publish the proofs. 


$$
S \mid T \rightarrow \epsilon \in S+T \rightarrow S+T C_{+} F \rightarrow T \neq F-S .
$$

In other words, two separable nontrivial classes are not complementary.

TheOREM 4.2(5). If $A$ and $B$ are finite classes, the condition stated in Theorem 4.1 is also sufficient for $A \mid B$.

Proof. Let $A=\left[\alpha_{0}, \cdots, \alpha_{i}\right]$ and $B=\left[\beta_{0}, \cdots, \beta_{k}\right]$ satisfy the condition mentioned in Theorem 4.1. Suppose $x \leqq i, y \leqq k$; then both $\alpha_{x} \subset \beta_{y}$ and $\beta_{y} \subset \alpha_{x}$ are false, hence both $\alpha_{x} \beta_{y}^{\prime}$ and $\beta_{y} \alpha_{x}^{\prime}$ are nonempty. Consequently, for every $x \leqq i$ and $y \leqq k$ there exist numbers $a_{x y}$ and $b_{y x}$ such that $a_{x y} \in \alpha_{x} \beta_{y}^{\prime}$ and $b_{y x} \in \beta_{y} \alpha_{x}^{\prime}$. Let $\Gamma_{a}=\left\|a_{x y}\right\|$ and $\Gamma_{b}=\left\|b_{y x}\right\|$. Put

$$
C=\sum_{n=0}^{i} T\left(\alpha_{n}\right)+\sum_{n=0}^{k} T\left(\beta_{n}\right) .
$$

Let $\gamma=\theta C$, then $\gamma$ is r.e. For every $n \in \gamma$ the set $\omega_{n}$ contains at least one row of $\Gamma_{a}$ or at least one row of $\Gamma_{b}$ (possibly both). For every $n \in \gamma$ we generate $\omega_{n}$, i.e., we start computing $q_{n}(x)$ for $x \in \delta q_{n}$ until we have obtained all elements in some row of $\Gamma_{a}$ or all elements in some row of $\Gamma_{b}$. If a complete row of $\Gamma_{a}$ is obtained first we put $n$ in $\gamma_{1}$, if a complete row of $\Gamma_{b}$ is obtained first we put $n$ in $\gamma_{2}$; if both these events happen simultaneously we favor $\gamma_{1}$ by putting $n$ in $\gamma_{1}$. Thus $\gamma_{1}$ and $\gamma_{2}$ are two disjoint r.e. sets and $\theta A+\theta B C \gamma_{1}+\gamma_{2}$ $=\gamma$. Suppose $n \in \theta A$. Then $n \in \gamma, \omega_{n} \in A, \omega_{n}$ includes no row of $\Gamma_{b}$ and $n \notin \gamma_{2}$. Thus $n \in \gamma-\gamma_{2}=\gamma_{1}$; hence $\theta A \subset \gamma_{1}$. Similarly it is seen that $\theta B \subset \gamma_{2}$. Consequently, $A \mid B$.

TheOREM 4.3(5). If $A$ and $B$ are infinite classes, the condition stated in Theorem 4.1 is not sufficient for $A \mid B$.

Proof. Let $\sigma \in F-E, A_{\sigma}=\{(n) \mid n \in \sigma\}, B_{\sigma}=\{(n) \mid n \notin \sigma\}$. We have seen in the beginning of this section that $A_{\sigma}$ is inseparable from $B_{\sigma}$. Nevertheless, neither of the classes $A_{\sigma}$ and $B_{\sigma}$ contains a set which is included in a set belonging to the other one.

THEOREM 4.4. The following condition is sufficient for $A \mid B$ : there exist two arrays $\left\{\alpha_{n}\right\}$ and $\left\{\beta_{n}\right\}$ such that

(1) every member of $A$ includes at least one row of $\left\{\alpha_{n}\right\}$ and every member of $B$ includes at least one row of $\left\{\beta_{n}\right\}$,

(2) no member of $A$ includes a row of $\left\{\beta_{n}\right\}$ and no member of $B$ includes $a$ row of $\left\{\alpha_{n}\right\}$.

Proof. Let the classes $A$ and $B$ and the arrays $\left\{\alpha_{n}\right\}$ and $\left\{\beta_{n}\right\}$ satisfy the conditions (1) and (2). Put

$$
C=\sum_{n=0}^{\infty} T\left(\alpha_{n}\right)+\sum_{n=0}^{\infty} T\left(\beta_{n}\right) .
$$


The set $\gamma=\theta C$ is r.e. We define $\gamma_{1}$ and $\gamma_{2}$ as in the proof of Theorem 4.2 with the slight modification that ' $\alpha_{0}, \cdots, \alpha_{i}$ ' and ' $\beta_{0}, \cdots, \beta_{k}$ ' are replaced by ' $\alpha_{0}, \alpha_{1}, \ldots$ ' and ' $\beta_{0}, \beta_{1}, \ldots$ ' respectively. We again have $\theta A+\theta B C \gamma_{1}+\gamma_{2}$ $=\gamma$, where $\gamma_{1}$ and $\gamma_{2}$ are disjoint r.e. sets. Suppose $n \in \theta A$. Then $n \in \gamma, \omega_{n} \in A$, $n \notin \gamma_{2}$ by (2) and hence $n \in \gamma-\gamma_{2}=\gamma_{1}$. Thus $\theta A \subset \gamma_{1}$; similarly $\theta B \subset \gamma_{2}$. We conclude that $A \mid B$.

COROLlary. There are exactly $c$ pairs of separable classes.

Proof. $F$ has only $c$ pairs of subclasses, since $F$ is denumerable. We proceed to show that there are at least $c$ pairs of separable classes. Let $\delta$ stand for the set of all even numbers and $\eta=\delta^{\prime}$. Put

$$
U=\{\sigma \mid \sigma \in F-[0] \& \sigma \subset \delta\}, \quad W=\{\sigma \mid \sigma \in F-[0] \& \sigma \subset \eta\} .
$$

The classes $U$ and $W$ satisfy the sufficient condition stated in Theorem 4.4 with respect to the arrays $\left\{\alpha_{n}\right\}=\{(2 n)\}$ and $\left\{\beta_{n}\right\}=\{(2 n+1)\}$, hence $U \mid W$. Let $U_{1}$ and $W_{1}$ form any pair of classes such that $U_{1} \subset U$ and $W_{1} \subset W$, then $U_{1} \mid W_{1}$. The pair $\left(U_{1}, W_{1}\right)$ can be chosen in $c^{2}=c$ different ways, because both $U$ and $W$ are denumerable. The desired result follows.

It should be pointed out that neither of two separable, nontrivial classes need contain any finite sets. This is clear if one considers $A=U \cdot(F-Q)$ and $B=W \cdot(F-Q)$, where $U$ and $W$ are the classes defined above. In this example both $A$ and $B$ are infinite classes.

Theorem $4.5\left(^{6}\right)$. (Added October 21,1957 .) The condition stated in Theorem 4.4 is not necessary for $A \mid B$.

Proof. With every partial recursive function $f(x)$ we associate

$$
\gamma_{f}=\{j(x, f(x)) \mid x \in \delta f\}, \quad m(f)=(\mu x)\left(f=q_{x}\right) .
$$

Let $c(x)$ denote the recursive function which is identically zero. Put

$$
A=\left\{\gamma_{c}\right\}, \quad B=\left\{\gamma_{f} \mid f \text { is recursive \& }(\exists y)(y \leqq m(f) \& f(y)>0)\right\} .
$$

We claim:

(1) $A \mid B$, (2) $A$ and $B$ do not satisfy the condition stated in Theorem 4.4.

Re (1). For every partial recursive function $f(x)$ the function $f(x)$ can be effectively reconstructed from $\gamma_{f}$. Thus there exists a partial recursive function $h(n)$ such that

$$
f \text { partial recursive \& } \gamma_{f}=\omega_{n} \rightarrow h(n) \text { defined \& } f=q_{h(n)} .
$$

Let $\eta$ be the set of all $n$ such that $g_{h(n)}$ is defined at $0,1, \cdots, h(n)$, then $\eta$ is r.e. We define for $n \in \eta$,

$$
d(n)=\left\{\begin{array}{l}
(\mu y)\left[y \leqq h(n) \& q_{h(n)}(y)>0\right] \text { if }(\exists y)\left[y \leqq h(n) \& q_{h(n)}(y)>0\right], \\
0, \text { otherwise. }
\end{array}\right.
$$

( ${ }^{8}$ We are indebted for this theorem to Mrs. Pour-El. 
Thus $d(n)$ is a partial recursive function with $\eta$ as domain and

$$
\omega_{x} \in A+B \rightarrow q_{h(x)} \text { recursive } \rightarrow d(x) \text { defined. }
$$

We now have:

$$
\begin{aligned}
\omega_{x} \in A & \rightarrow \omega_{x}=\gamma_{c} \rightarrow q_{h(x)}=0 \rightarrow q_{h(x)} d(x)=0, \\
\omega_{x} \in B & \rightarrow q_{h(x)} \text { recursive \& }(\exists y)\left[y \leqq \mathrm{~m}\left(q_{h(x)}\right) \& q_{h(x)}(y)>0\right] \\
& \rightarrow(\exists y)\left[y \leqq h(x) \& q_{h(x)}(y)>0\right] \\
& \rightarrow q_{h(x)} d(x)>0 .
\end{aligned}
$$

Thus $A \mid B$.

$\operatorname{Re}(2)$. We first prove the lemma: for each number $n$ there exists a function $f(x)$ such that

$$
\gamma_{f} \in B \&(\forall x)[x \leqq n \rightarrow f(x)=0] .
$$

Suppose namely that $B$ is the family of all recursive functions $g$ such that

$$
g(x)=0 \text { for } x \leqq n \& g(x)>0 \text { for } x>n .
$$

Then $B$ is a denumerable family and for $g_{1}, g_{2} \in B$ we have: $g_{1} \neq g_{2}$ implies $m\left(g_{1}\right) \neq m\left(g_{2}\right)$. The set $\{m(g) \mid g \in Q\}$ is therefore infinite; thus it must contain a number greater than $n$. Thus, for some $f \in B$ we have $m(f)>n$, hence $\gamma_{f} \in B$ and $f(x)=0$ for $x \leqq n$. This proves the lemma. Now suppose there were an array $\left\{\alpha_{n}\right\}$ such that $\gamma_{c}$ included at least one row of that array, say $\alpha_{t}$. Since

$$
\gamma_{c}=(j(0,0), j(1,0), j(2,0), \cdots)
$$

there must be numbers $x_{0}, \cdots, x_{r}$ such that

$$
\alpha_{t}=\left(j\left(x_{0}, 0\right), j\left(x_{1}, 0\right), \cdots, j\left(x_{r}, 0\right)\right) .
$$

Using the lemma there is a function $f \in B$ such that $\gamma_{f} \in B$ and $f\left(x_{i}\right)=0$ for $0 \leqq i \leqq r$. This implies, however, $\gamma_{f} \supset \alpha_{i}$, contrary to the assumption that no set in $B$ includes a row of $\left\{\alpha_{n}\right\}$.

We note for future use that the argument employed in the last proof establishes more than we set out to prove. For the fact that $\left\{\alpha_{n}\right\}$ was an array was never used. For the classes $A$ and $B$ defined above we have therefore established the following stronger statement: there is no class $C$ of finite sets such that $\sigma \in A+B \rightarrow[\sigma \in A \leftrightarrow(\exists \gamma)(\gamma \in C \& \gamma \subset \sigma)]$.

$\mathrm{V}$. Topological considerations. We recall that a class $A$ is a $\Sigma T$-class if $A=O$ or $A=\sum_{0}^{\infty} T\left(\alpha_{n}\right)$ for some sequence $\left\{\alpha_{n}\right\}$ of finite sets.

PROPOSITION 5.1. The sum of arbitrarily many $\Sigma T$-classes is again a $\Sigma T$-class. The product of finitely many $\Sigma T$-classes is again a $\Sigma T$-class.

Proof. We shall show that the product of two $\Sigma T$-classes is again a $\Sigma T$ - 
class. Using this fact the reader will readily complete the proof. Let $A$ and $B$ be $\Sigma T$-classes. Disregarding the trivial case that either $A$ or $B$ is empty we have

$$
A=\sum_{0}^{\infty} T\left(\alpha_{n}\right), \quad B=\sum_{0}^{\infty} T\left(\beta_{n}\right),
$$

for two sequences $\left\{\alpha_{n}\right\}$ and $\left\{\beta_{n}\right\}$ of finite sets. Thus

$$
\begin{aligned}
A \cdot B & =\sum_{0}^{\infty} T\left(\alpha_{n}\right) \cdot \sum_{0}^{\infty} T\left(\beta_{n}\right)=\sum_{0}^{\infty} T\left(\alpha_{k(n)}\right) \cdot T\left(\beta_{l(n)}\right) \\
& =\sum_{0}^{\infty} T\left(\alpha_{k(n)}+\beta_{l(n)}\right) .
\end{aligned}
$$

Definitions. A class is open if it is a $\Sigma T$-class. The class $S$ is a neighborhood if $S=T(\sigma)$ for some finite set $\sigma$. A neighborhood of the r.e. set $\alpha$ is any neighborhood which contains $\alpha$.

Note that $T(\sigma)$ is a neighborhood of $\alpha$ if and only if $\sigma$ is a finite subset of $\alpha$. By Proposition 5.1 we have imposed a topology on the class $F$ by defining the open classes as the $\Sigma T$-classes. The neighborhood system which we defined evidently yields this topology.

Considered as a topological space $F$ is connected, since every nonempty open class contains $\epsilon$. Of the five separation axioms $T_{0}, \cdots, T_{4}$ the class $F$ satisfies only the first one. Suppose namely that $\alpha, \beta \in F, \alpha \neq \beta$ and $n \in \alpha \beta^{\prime}$ $+\alpha^{\prime} \beta$. Then either $n \in \alpha \beta^{\prime}$ or $n \in \alpha^{\prime} \beta$. Thus, either $L(n)$ is a neighborhood of $\alpha$ which does not contain $\beta$ or $L(n)$ is a neighborhood of $\beta$ which does not contain $\alpha$; this shows that $F$ is a $T_{0}$-space. To show that $F$ is not a $T_{1}$-space we observe that the class $F-[\epsilon]$ is not open, since it does not contain $\epsilon$. We conclude that the class $[\epsilon]$ is not closed.

Definition. An effective operation on $F$ is a function $\Phi$ from $F$ into itself such that $\Phi\left(\omega_{n}\right)=\omega_{f(n)}$ for some recursive function $f(n)$.

THEOREM 5.1. (1) Every c.r.e. class is open, but not conversely.

(2) Every effective operation on $F$ is continuous, but not conversely.

Proof. (1) Every c.r.e. class is open by Corollary 2 of Theorem 3.1. On the other hand there are $c$ open classes of which only denumerably many are c.r.e. (see the remark after Proposition 1.8).

(2) Let $\Phi$ be an effective operation on $F$. By $[14$, p. 313, Corollary B] we know that

$$
\Phi\left(\omega_{n}\right)=\left\{x \mid(\exists y)\left[\rho_{g(x, y)} \subset \omega_{n}\right]\right\},
$$

for some partial recursive function $g(x, y)$, where the relation between the brackets is considered false in case $g(x, y)$ is undefined. Let $V$ be a neighbor- 
hood of $\Phi\left(\omega_{n}\right)$. To establish the continuity of $\Phi$ at $\omega_{n}$ we show that there is a neighborhood $U$ of $\omega_{n}$ such that $\Phi(U) \subset V$. Let $\alpha$ be the finite set such that $V=T(\alpha)$. If $\alpha=o$ we have $V=F$ and we can take $U=T(o)=F$. Let $\alpha \neq 0$, say $\alpha=(a(0), \cdots, a(r))$. From now on the range of $i$ will be assumed to be $0, \cdots, r$. Since $\alpha \subset \Phi\left(\omega_{n}\right)$ there is for every $i$ a $y$ such that $\rho_{o(a(i), y)} \subset \omega_{n}$, say $y(i)$. We define

$$
\beta=\sum_{i} \rho_{g(a(i), v(i))}
$$

Thus $\beta C \omega_{n}$ and $\omega_{n}$ belongs to $T(\beta)$. Put $U=T(\beta)$. We have

$$
\begin{aligned}
\omega_{k} \in U & \rightarrow \beta \subset \omega_{k} \\
& \rightarrow \rho_{o(a(i), v(i))} \subset \omega_{k} \text { for every } i . \\
& \rightarrow a(i) \in \Phi\left(\omega_{k}\right) \text { for every } i \\
& \rightarrow \alpha \subset \Phi\left(\omega_{k}\right) \\
& \rightarrow \Phi\left(\omega_{k}\right) \in T(\alpha)=V .
\end{aligned}
$$

It follows that $\Phi(U) \subset V$. Thus $\Phi$ is continuous at every point $\omega_{n}$ of $F$. It remains to be proved that there are continuous mappings of $F$ into itself which are not effective operations. Since there are only denumerably many effective operations on $F$, it suffices to construct $c$ continuous mappings of $F$ into itself. We do this by associating with every set $\gamma$ a mapping $\Phi_{\gamma}$ defined by

$$
\Phi_{\gamma}(\sigma)=\left\{\begin{array}{l}
\epsilon \text { if } \sigma \gamma \neq 0, \\
0 \text { if } \sigma \gamma=0 .
\end{array}\right.
$$

$\Phi_{\gamma}$ maps $F$ into itself for every $\gamma$ and $\gamma$ may be chosen in $c$ different ways. Let $\gamma \neq \delta, n \in \gamma \delta^{\prime}+\gamma^{\prime} \delta$ and $\alpha=(n)$. In case $n \in \gamma \delta^{\prime}$ (in case $n \in \gamma^{\prime} \delta$ ) the set $\alpha$ is mapped onto $\epsilon$ (respectively o) by $\Phi_{\gamma}$, but onto o (respectively $\epsilon$ ) by $\Phi_{\delta}$. This implies that different choices of $\gamma$ yield different mappings $\Phi_{\gamma}$. We now show that $\Phi_{\gamma}$ is continuous for every choice of $\gamma$. Let $V=T(\alpha)$ be a neighborhood of $\Phi_{\gamma}\left(\omega_{n}\right)$. We wish to find a neighborhood $U$ of $\omega_{n}$ such that $\Phi_{\gamma}(U) \subset V$. If $\omega_{n} \cdot \gamma=0$ we can take any neighborhood of $\omega_{n}$ for $U$, since in that case $\Phi_{\gamma}\left(\omega_{n}\right)$ $=o, \alpha=o, V=F$. Now suppose $\omega_{n} \cdot \gamma \neq 0$. We take $U=L(x)$ for some $x$ in $\omega_{n} \cdot \gamma$. Then

$$
\begin{aligned}
\omega_{k} \in U & \rightarrow x \in \omega_{k} \rightarrow \omega_{k} \cdot \gamma \neq 0 \\
& \rightarrow \Phi_{\gamma}\left(\omega_{k}\right)=\epsilon \in V .
\end{aligned}
$$

Let us call the classes $A$ and $B$ separated if they are disjoint and there are open classes $A_{1}$ and $B_{1}$ such that $A=A_{1}(A+B)$ and $B=B_{1}(A+B)$. We can prove that $A \mid B$ does not imply that $A$ and $B$ are separated. For let $A$ and $B$ be defined as in the proof of Theorem 4.5. Then $A \mid B$; if $A$ and $B$ were separated there would be an open class 


$$
A_{1}=\sum_{0}^{\infty} T\left(\alpha_{n}\right)
$$

such that $A=A_{1}(A+B)$. Thus

$$
\sigma \in A+B \rightarrow\left[\sigma \in A \leftrightarrow(\exists n)\left(\alpha_{n} \subset \sigma\right)\right]
$$

contrary to the remark made after the proof of Theorem 4.5.

Using the second part of Theorem 5.1 we can give another proof of Theorem 2.1. Let $S \neq O, S \neq F$ and

$$
\Phi\left(\omega_{n}\right)= \begin{cases}(0) & \text { for } \omega_{n} \in S, \\ (1) & \text { for } \omega_{n} \in F-S .\end{cases}
$$

We wish to prove that $S$ is undecidable. Suppose $S$ were decidable. Then $\theta S$ would be a recursive set and $\Phi$ an effective and therefore continuous operation on $F$. We now have a contradiction. For on the one hand $\Phi(F)=[(0),(1)]$ must be connected since $F$ is connected; on the other hand, $[(0)]=L(0) \cdot \Phi(F)$ and $[(1)]=L(1) \cdot \Phi(F)$, hence $\Phi(F)$ is disconnected. We conclude that $S$ must be undecidable.

VI. Productive classes. Let $A$ be a class which is not r.e. For every r.e. subclass $B$ of $A$ there now exists a set $\gamma \in A-B$, i.e., a witness to the fact that $B$ is properly included in $A$. If, given any recursive function $f_{n}(x)$ which generates the r.e. subclass $B$ of $A$ such a witness can be effectively found, we call the class $A$ productive. We wish to show that many classes are productive, e.g., $F-E, F-Q, E-Q$ and the class of all creative sets.

An infinite set which has no infinite r.e. subset is called immune. A r.e. set is simple, if its complement is immune; a r.e. set is hypersimple, if it has an infinite complement and includes at least one row of every discrete array. A set in $F-E$ is called mesoic if its complement is neither immune nor productive.

Notations.

$D=E-(P+Q)$,

$H=$ class of all creative sets,

$Z=$ class of all simple sets,

$Z_{0}=$ class of all hypersimple sets,

$J=$ class of all mesoic sets.

It was proved by Post [15] that $H$ and $Z_{0}$ are nonempty and that $Z_{0} C_{+} Z$; it was shown in [3] that $J$ is nonempty. Thus

$$
E=Q+D+P, \quad F-E=H+J+Z,
$$

where in each of these equalities the three classes on the right side are nonempty and mutually disjoint.

The subset $\pi$ of the productive set $\alpha$ is called a productive center of $\alpha$, if 
$\pi=\left\{p(n) \mid \omega_{n} \subset \alpha\right\}$, for some productive function $p(n)$ of $\alpha\left(^{7}\right)$. The set $\alpha$ is called semiproductive relative to the partial recursive function $p(n)$ if

$$
\omega_{n} \subset \alpha \rightarrow\left\{\begin{array}{l}
(1) p(n) \text { is defined, } \\
(2) \omega_{n} \subset_{+} \omega_{p(n)} \subset \alpha .
\end{array}\right.
$$

Every partial recursive function $p(n)$ related to $\alpha$ by (1) is called a semiproductive function of $\alpha$. The set $\alpha$ is semiproductive if it has at least one semiproductive function. It is readily seen that every productive set is semiproductive; the converse is false by $[4, \mathrm{~T} 4.2]$.

We recall from $\S \mathrm{I}$ that the class $A$ is r.e. if and only if $A=\left\{\omega_{x} \mid x \in \omega_{n}\right\}$ for some $n$.

Notation. $W_{n}=\left\{\omega_{x} \mid x \in \omega_{n}\right\}$.

It follows that $\left\{W_{n}\right\}$ is a r.e. sequence of r.e. classes in which every r.e. class occurs infinitely many times; $W_{0}=O$, since $\omega_{0}=0$. Every number $n$ such that $A=W_{n}$ is an index of the r.e. class $A$. We say that a r.e. class is given if one of its indices is given. It is clear from the proof of Proposition 1.5 that given an index of any r.e. class $A$ which contains at least one nonempty set we can effectively find a recursive function $f_{n}(x)$ which generates the class of all nonempty members of $A$.

Definition. The class $A$ is called productive relative to the partial recursive function $p(n)$, if

$$
W_{n} \subset A \rightarrow \begin{cases}(1) & p(n) \text { is defined, } \\ (2) & \omega_{p(n)} \in A-W_{n} .\end{cases}
$$

Let $p(n)$ be a partial recursive function related to $A$ by (1). Then $p(n)$ is called a productive function of $A$ and the class $\left\{\omega_{p(n)} \mid W_{n} \subset A\right\}$ is called the productive center of $A$ relative to $p(n)$ [written ' $C(A, p)$ ']. The class $A$ is called productive if it has at least one productive function. The subclass $C$ of the productive class $A$ is called a productive center of $A$, if $C=C(A, p)$ for some productive function $p(n)$ of $A$.

We now list some elementary properties of productive classes. The proofs are similar to those of the corresponding properties of productive sets established in [4] and are therefore omitted.

Propositions.

6.1. A productive class is not r.e., but has an infinite r.e. subclass [cf. 4, $\mathrm{T}$ 2.2].

6.2. The class $A$ is productive if and only if $A-[0] \neq O$ and there exists $a$ partial recursive function $q(n)$ such that

(7) The following unpublished result is due to Mrs. C. Karp: among the productive centers of any productive set there are denumerably many which are mutually disjoint. 


$$
\left.\begin{array}{r}
W_{n}-[0] \neq O \\
W_{n} \subset A
\end{array}\right\} \rightarrow\left\{\begin{array}{ll}
(1) & q(n) \text { is defined, } \\
(2) & \omega_{q(n)} \in A-W_{n} .
\end{array} \quad[\text { cf. 4, Proposition B], }\right.
$$

6.3. The class $A$ is productive if and only if there exists a partial recursive function $t(n)$ such that

$$
W_{n} \subset A \rightarrow\left\{\begin{array}{l}
(1) t(n) \text { is defined, } \\
\text { (2) } W_{t(n)} \subset A-W_{n}, \\
(3) W_{t(n)} \text { is infinite. }
\end{array} \quad\right. \text { [cf. 4, Proposition C], }
$$

6.4. If $C_{0}$ is a productive center of the productive class $A$, there is a productive center $C_{1}$ of $A$ such that $C_{1} \subset C_{0}$ and $C_{0}-C_{1}$ is infinite [cf. 4, Proposition D].

Corollary. Every productive class $A$ has a productive center $C_{1}$ such that $A-C_{1}$ is infinite.

6.5. Every productive class $A$ has exactly denumerably many productive centers and exactly denumerably many productive functions [cf. 4, $T 2.3$ ].

6.6. Let $A$ be productive relative to $p(n)$ and $C(A, p) \subset B \subset A$. Then $B$ is also productive relative to $p(n)$ and $C(B, p) \subset C(A, p)[c f .4, T 2.4]$.

Corollary 1. If $A$ is productive relative to $p(n)$, so is $C(A, p)$.

COROLlary 2. Every productive class includes exactly c productive classes.

6.7. Let $A$ be a productive class with a productive center $C$ and let $B$ be a r.e. class. Then in $C$,

(1) $B C A$ implies that $A-B$ is productive with a productive center included

(2) $A \subset B$ implies that $A+(F-B)$ is productive with a productive center included in $C$ [cf. 4, T. 2.5].

THEOREM 6.1. Every productive class has a recursive productive function.

Proof. Let $p(n)$ be one of the productive functions of the productive class $A$. We now use the part of the proof of Proposition 1.11 which starts with "There exists" and ends with "Since $g(x)$, " but omitting (3). We then proceed as follows.

$$
\begin{aligned}
& \left(1^{*}\right) n \in \delta p \rightarrow I W_{f(n)}=W_{n}+\left[\omega_{p(n)}\right], \\
& \left(2^{*}\right) n \in \delta p \rightarrow W_{f(n)}=O \subset A, \\
& \left(3^{*}\right) n \in \delta p \rightarrow p f(n) \text { is defined. }
\end{aligned}
$$

We claim that $p f(x)$ is also a productive function of $A$. For by $\left(1^{*}\right)$

$$
W_{x} \subset A \rightarrow W_{f(x)} \subset A \rightarrow \omega_{p f(x)} \in A-\left(W_{x}+\left[\omega_{p(x)}\right]\right) \rightarrow \omega_{p f(x)} \in A-W_{x} .
$$

Since both $p(x)$ and $p f(x)$ are productive functions of $A$, so is the recursive function $g(x)$. 
TheORem 6.2. (1) If $A$ is a productive class with a productive center $C$, then $\theta A$ is a productive set with a productive center included in $\theta C$.

(2) If $\theta A$ is a productive set, $A$ is not necessarily a productive class.

Proof. (1) Let $A$ be productive relative to $p(n)$ and let $C=C(A, p)$. Then $\omega_{n} \subset \theta A \rightarrow W_{n} \subset A \rightarrow \omega_{p(n)} \in\left(A-W_{n}\right) \cdot C \rightarrow p(n) \in\left(\theta A-\omega_{n}\right) \cdot \theta C$.

(2) $Q$ is not a $\Sigma T$-class, hence $\theta Q$ is a productive set. Nevertheless, $Q$ is not a productive class, since it is r.e.

Definition. The class $A$ is called semiproductive relative to the partial recursive function $p(n)$ if

$$
W_{n} \subset A \rightarrow \begin{cases}(1) & p(n) \text { is defined, } \\ (2) & W_{n} C_{+} W_{p(n)} \subset A .\end{cases}
$$

Every partial recursive function $p(n)$ related to $A$ by (1) is called a semiproductive function of $A$. A class is semiproductive if it has at least one semiproductive function.

Every productive class is semiproductive; the converse is false, for if $\alpha$ is a semiproductive, but not productive set [see 4, T 4.2] the class $\{(n) \mid n \in \alpha\}$ is also semiproductive, but not productive.

THEOREM 6.3. The following three conditions are equivalent: $\alpha$ is semiproductive, $K(\alpha)$ is semiproductive, $K(\alpha)$ is productive.

Proof. There exist recursive functions $q(n)$ and $t(n)$ such that

$$
\begin{aligned}
\omega_{q(n)} & =\Sigma \omega_{x}, \text { where } x \text { ranges over } \omega_{n} \text { (hence } \omega_{x} \text { over } W_{n} \text { ), } \\
W_{\ell(n)} & =K\left(\omega_{n}\right) .
\end{aligned}
$$

(1) If $K(\alpha)$ is productive it is semiproductive. To establish the converse, assume that $K(\alpha)$ is semiproductive relative to $p(n)$.

$$
\begin{aligned}
W_{n} \subset K(\alpha) & \rightarrow \omega_{q(n)} \subset \alpha \\
& \rightarrow W_{n} \subset K\left(\omega_{q(n)}\right)=W_{t q(n)} \subset K(\alpha) \\
& \rightarrow W_{n} \subset W_{t q(n)} \subset+W_{p t q(n)} \subset K(\alpha) \\
& \rightarrow \omega_{q p t q(n)} \in K(\alpha)-W_{n .}
\end{aligned}
$$

Thus $K(\alpha)$ is productive relative to $q p t q(n)$.

(2) Suppose $\alpha$ is semiproductive relative to $p(n)$.

$$
\begin{aligned}
W_{n} \subset K(\alpha) & \rightarrow \omega_{q(n)} \subset \alpha \\
& \rightarrow \omega_{q(n)} \subset+\omega_{p q(n)} \subset \alpha \\
& \rightarrow \omega_{p q(n)} \in K(\alpha)-W_{n} .
\end{aligned}
$$

Thus $K(\alpha)$ is productive relative to $p q(n)$. Conversely, assume that $K(\alpha)$ is productive relative to $p(n)$. 


$$
\begin{aligned}
\omega_{n} \subset \alpha & \rightarrow W_{t(n)} \subset K(\alpha) \\
& \rightarrow \omega_{p t(n)} \in K(\alpha)-W_{t(n)} \\
& \rightarrow \omega_{p t(n)} \subset \alpha \& \sim\left(\omega_{p t(n)} \subset \omega_{n}\right) .
\end{aligned}
$$

It follows that $\alpha$ is semiproductive relative to any partial recursive function $a(n)$ such that $\omega_{a(n)}=\omega_{n}+\omega_{p t(n)}$.

Combining the last theorem with the fact that not every semiproductive set is productive we conclude that if the set $\alpha$ is productive, so is the class $K(\alpha)$, but not conversely.

Notation. $\operatorname{Im}(\alpha)=\{\sigma \mid \sigma \cong \alpha\}$.

THEOREM 6.4. $F-Q$ is a productive class which for every $\alpha \in(F-Q)$ $-(Z+P)$ has a productive center which is included in $\operatorname{Im}(\alpha)$.

Proof. Let $W_{n}-[0]$ be nonempty. From $n$ we can effectively obtain a recursive function $f_{m}^{(n)}(x)$ of $m$ and $x$ which generates $W_{n}-[0]$. For the sake of simplicity we shall omit the superscript ' $(n)$ ' and write ' $f_{m}(x)$ '. One should, however, keep in mind that all functions and sets defined in terms of $f_{m}(x)$ depend on $n$. Let the sequence $f_{m}(0), f_{m}(1), \cdots$ be called row $m$. We define $c(0)=f_{0}(0)$,

$d(0)=$ the first element in row 0 which differs from $c(0)$, $c(x+1)=$ the first element in row $x+1$ which exceeds

$$
\max (c(0), d(0), \cdots, c(x), d(x)),
$$

$d(x+1)=$ the second such element in row $x+1$,

$c^{\prime}(0)=$ the smallest number not occurring in $\{c(n)\}$ which is smaller than some number in $\{c(n)\}$,

$c^{\prime}(x+1)=$ the smallest number not occurring in $\{c(n)\}$ which exceeds $c^{\prime}(x)$, but is smaller than some number in $\{c(n)\}$.

We denote the ranges of the functions $c(x), d(x)$ and $c^{\prime}(x)$ by $\gamma, \delta$ and $\eta$ respectively. The functions $c(x), d(x)$ and $c^{\prime}(x)$ are clearly partial recursive; whether they are recursive depends on the number $n$. In the special case that $W_{n} \subset F-Q$ we have: $c(x), d(x), c^{\prime}(x)$ are recursive and strictly increasing, $\eta=\gamma^{\prime}$, and $\gamma$ is not included in any set of $W_{n}$ [cf. 2, the proof of T 1.3]. In this special case we have therefore $\gamma \in(F-Q)-W_{n}$; thus, $F-Q$ is a productive class. Let $\alpha \in(F-Q)-(Z+P)$. We wish to prove that $F-Q$ has a productive center which is included in $\operatorname{Im}(\alpha)$. The set $\alpha$ is included in some set in $D$, say $\tau$. Let $t(x)$ and $t^{\prime}(x)$ denote the strictly increasing recursive functions which range over $\tau$ and $\tau^{\prime}$ respectively. Let $g(x)$ be the partial recursive function which maps $t(x)$ onto $c(x)$, whenever $c(x)$ is defined and $t^{\prime}(x)$ onto $c^{\prime}(x)$, whenever $c^{\prime}(x)$ is defined. Put $\gamma_{\alpha}=g(\alpha)$. In the special case $W_{n} \subset F-Q$ we have: $g(x)$ is a recursive permutation, $\gamma_{\alpha} \cong \alpha, \gamma_{\alpha}=g(\alpha) \subset g(\tau)=\gamma$. Hence $\eta \subset \gamma_{\alpha}^{\prime}$, and therefore $\gamma_{\alpha}$ is not included in any set of $W_{n}$. Thus,

$$
\gamma_{\alpha} \in(F-Q)-W_{n} \& \gamma_{\alpha} \in \operatorname{Im}(\alpha) \text {. }
$$


This completes the proof.

Corollary. Let $S$ be one of the classes $D, H$ and $J$. Then $S$ is productive and for every $\alpha \in S$ it has a productive center included in $\operatorname{Im}(\alpha)$.

Proof. Under the hypothesis $F-Q$ has a productive center $C_{\alpha}$ such that

$$
C_{\alpha} \subset \operatorname{Im}(\alpha) \subset S \subset F-Q .
$$

We now apply Proposition 6.6.

The class $A$ is recursively closed if $\alpha \in A$ implies $\operatorname{Im}(\alpha) \subset A$. The class $S$ is called a product system if $S$ is closed under the operation of intersection. We say that $\alpha$ is almost included in $\beta$ (written ' $\alpha \subset^{*} \beta^{\prime}$ ) if $\alpha \beta^{\prime}$ is finite.

Theorem 6.5. Suppose (1) $O C_{+} A \subset F-Q$, (2) $A$ is recursively closed, (3) $A \subset T \subset F-Q$ for some product system $T$, (4) $A$ is not included in $P$. Then $A$ is a productive class which for every $\alpha \in A-P$ has a productive center which is included in $\operatorname{Im}(\alpha)$.

Proof. Let $W_{n}-[0]$ be nonempty. From $n$ we can effectively obtain a recursive function $f_{m}(x)$ (depending on $n$ ) of $m$ and $x$ which generates $W_{n}$ $-[o]$. Put $\alpha_{m}=\rho f_{m}$ and denote the product of the $i+1$ sets $\alpha_{0}, \cdots, \alpha_{i}$ by $\beta_{i}$. From $f_{m}(x)$ we can effectively find a partial recursive function $b_{i}(x)$ such that $\beta_{i}=\rho b_{i}$. We define

$$
\begin{aligned}
& c(0)=b_{0}(0), \\
& c(x+1)=b_{x+1}(\mu y)\left[b_{x+1}(y)>c(x)\right], \\
& \gamma=(c(0), c(2), \cdots),
\end{aligned}
$$

$c^{\prime}(0)=$ the smallest number not occurring in $\{c(2 n)\}$ which is smaller than some number in $\{c(2 n)\}$,

$c^{\prime}(x+1)=$ the smallest number not occurring in $\{c(2 n)\}$ which exceeds $c^{\prime}(x)$, but is smaller than some number in $\{c(2 n)\}$.

The functions $c(x)$ and $c^{\prime}(x)$ are partial recursive. In the special case $W_{n} \subset A$ we have: $c(x)$ and $c^{\prime}(x)$ are recursive and strictly increasing, $\gamma \in D$, $\gamma^{\prime}=\rho c^{\prime}$; moreover,

$$
\sigma \in W_{n} \rightarrow \gamma \subset^{*} \sigma
$$

[cf. 2, the proof of T 3.5]. Suppose $\alpha \in A-P$ and $\tau$ is a subset of $\alpha$ which belongs to $D$. Let $t(x)$ and $t^{\prime}(x)$ be the strictly increasing recursive functions which range over $\tau$ and $\tau^{\prime}$ respectively. Let $h(x)$ be the partial recursive function which maps $t(x)$ onto $c^{\prime}(x)$ whenever $c^{\prime}(x)$ is defined, and $t^{\prime}(x)$ onto $c(x)$ whenever $c(x)$ is defined. Let us again restrict ourselves to the special case $W_{n} \subset A$. We then have: $h(x)$ is a recursive permutation, $h(\alpha) \cong \alpha$ and $\gamma^{\prime} \subset h(\alpha)$. If $h(\alpha)$ belonged to $W_{n}$ it would almost include $\gamma$ by (a). Since $\gamma^{\prime} \subset h(\alpha)$ this would imply the false statement $\alpha \in P$. Thus,

$$
h(\alpha) \in A-W_{n} \& h(\alpha) \in \operatorname{Im}(\alpha) .
$$

This completes the proof. 
Corollary. Let $S$ be one of the classes $Z, Z+P, Z_{0}, Z_{0}+P$. Then $S$ is productive and for every $\alpha \in S-P$ it has a productive center which is included in $\operatorname{Im}(\alpha)$.

Proof. Let $S$ satisfy the hypothesis. Then $S$ satisfies the conditions (1), (2) and (4) listed in Theorem 6.5. By [3, Theorems 1.5 and 1.6] the four classes involved are product systems. Thus $S$ is a productive class. For the part of the Corollary which deals with productive centers, see the proof of the Corollary of Theorem 6.4.

TheOREM 6.6. Let $\alpha \in F$. Then

(1) $\alpha \in P+Q \rightarrow \operatorname{Im}(\alpha)$ a r.e. class,

(2) $\alpha \notin P+Q \rightarrow \operatorname{Im}(\alpha)$ a productive class.

Proof. The first part is readily verified by the reader. Assume

$$
\alpha \in F-(P+Q)=D+H+J+Z \text {. }
$$

Let $S$ denote that one of the four classes $D, H, J, Z$ to which $\alpha$ belongs. Using the Corollaries of Theorems 6.4 and 6.5 we conclude that $S$ is a productive class with a productive center $C_{\alpha}$ such that $C_{\alpha} \subset \operatorname{Im}(\alpha) \subset S$. It follows by Proposition 6.6 that $\operatorname{Im}(\alpha)$ is a productive class.

Combining Theorems 6.4 and 6.5 with Propositions 6.6 and 6.7 one can establish the productivity of a large variety of classes.

In the remainder of this section we further restrict the meaning of the word "class" to that of "nonempty subclass of $F-[0]$ "; the letter ' $A$ ' will only be used to denote such a class. The information that a class $A$ is productive (or merely, that it is not r.e.) also tells us something about the family of all recursive functions which range over sets in $A$.

NOTATION. $\mathcal{O}_{n}=\left\{q_{y}(x) \mid y \in \omega_{n}\right\}$.

DEFINITION. Let $a$ be a family of recursive functions.

(1) $a$ is recursively enumerable if $a$ is empty or there exists a recursive function $b_{y}(x)$ of $x$ and $y$ such that

$$
f(x) \in Q \leftrightarrow(\exists y)\left[f(x)=b_{y}(x)\right]
$$

(2) $a$ is productive if there exists a partial recursive function $p(n)$ such that

$$
\mathcal{O}_{n} \subset Q \rightarrow\left\{\begin{array}{l}
(1) p(n) \text { is defined, } \\
(2) q_{p(n)}(x) \in Q-\mathcal{O}_{n} .
\end{array}\right.
$$

It is readily seen that the family $a$ of recursive functions is r.e. if and only if $a=O_{n}$ for some $n$. Also, while a productive family is never r.e., it always has an infinite r.e. subfamily. The notion of a productive center can be introduced for productive families in an obvious manner. The family of all primitive recursive functions of one variable is r.e. [7, p. 272] and the family of all recursive functions of one variable is productive [4, T 2.9 and its proof ]. 
Notations.

$\mathcal{F}=$ family of all recursive functions of one variable,

$\mathcal{F}_{1}=\{f \mid f \in \mathcal{F} \& f$ is one-to-one $\}$,

$\mathcal{F} A=\{f \mid f \in \mathcal{F} \& \rho f \in A\}$,

$\mathcal{F}_{1} A=\left\{f \mid f \in \mathcal{F}_{1} \& \rho f \in A\right\}$.

The following statements are readily verified:

(I) If the class $A$ is not r.e., the family $F A$ is not r.e., and any subfamily $B$ of $\mathcal{F} A$ such that $\{\rho f \mid f \in B\}=A$ is not r.e.,

(II) If the class $A$ is productive, the family $\mathcal{F} A$ is productive; if, moreover, $A \subset F-Q$, the family $\digamma_{1} A$ is also productive.

TheOREM 6.7. Let $S$ be any of the following classes: $F-E, F-Q, E-Q, D$, $H, Z, Z_{0}, J, \operatorname{Im}(\alpha)$ for any $\alpha \in F-(P+Q)$. Then both $F S$ and $\mathcal{F}_{1} S$ are productive families of recursive functions.

Proof. $F-E$ and $E-Q$ are productive, because $F-Q$ has a productive center included in $H$ and one included in $D$ and

$$
H \subset F-E \subset F-Q, \quad D \subset E-Q \subset F-Q \text {. }
$$

The other classes mentioned in the theorem are productive by previous theorems. The desired result now follows by (II).

VII. Recursively enumerable classes. In $\S \S I$ and VI we have seen that the following classes are r.e.:

(1) $O, F, E, P, Q$ and every finite class,

(2) $L(\alpha)$ and $K(\beta)$ for $\alpha, \beta \in F$,

(3) $\sum_{n=0}^{\infty} T\left(\alpha_{n}\right)$ for any array $\left\{\alpha_{n}\right\}$,

(4) $\operatorname{Im}(\alpha)$ for $\alpha \in P+Q$.

It was shown in $\S I I I$ that there are no c.r.e. classes besides those mentioned under (3). The r.e. classes $E, P, Q$ and $\operatorname{Im}(\alpha)$ for $\alpha \in P+Q$ are not $\Sigma T$-classes; they are therefore not c.r.e., though they are r.e. The class $L(\alpha)$ is r.e., but not c.r.e., if $\alpha \in F-Q$; the class $K(\beta)$ is r.e., but not c.r.e., if $\beta \in F-[\epsilon]$. It is not hard to show that the following classes are r.e.:

(5) $P \cdot L(\alpha)$ and $Q \cdot K(\beta)$ for $\alpha, \beta \in F$,

(6) $L(\alpha) \cdot K(\beta)=\{\sigma \mid \sigma \in F \& \alpha \subset \sigma \subset \beta\}$ for $\alpha, \beta \in F$.

The class mentioned under (6) is trivial if either $\alpha$ is not included in $\beta$ or both $\alpha=o$ and $\beta=\epsilon$.

We recall that the sum of two r.e. classes is a r.e. class and that the sum or product of two c.r.e. classes is a c.r.e. class.

TheOREM 7.1. There exist two r.e. classes whose product is not r.e.

Proof. Let $A=\{(n) \mid n \in \epsilon\}, B=\left\{\omega_{n} \mid n \in \omega_{n}\right\}$, then $A$ is r.e., because $\omega_{i(n)}=(n)$ for some recursive function $i(n)$, and $B$ is r.e., because $\left\{n \mid n \in \omega_{n}\right\}$ is r.e. Clearly, $A \cdot B=\left\{(n) \mid(n)=\omega_{n}\right\}$, hence

$$
(n) \in A \cdot B \leftrightarrow(n)=\omega_{n} .
$$


By the Corollary of Proposition 1.3 there are infinitely many numbers $k$ such that $(k)=\omega_{i(k)}=\omega_{k}$, hence $A \cdot B$ is infinite. Obviously $o \notin A \cdot B$. Suppose $A \cdot B$ were r.e., say

$$
A \cdot B=\left[\left(f_{0}(0)\right),\left(f_{1}(0)\right), \cdots\right]
$$

for some recursive function $f_{m}(x)$. Put $c_{m}=f_{m}(0), \gamma=\rho c$ then

$(n) \in A \cdot B \leftrightarrow n \in \gamma$, where $\gamma$ is r.e.

Combining (1) and (2) we get

$$
(n)=\omega_{n} \leftrightarrow n \in \gamma \text {, where } \gamma \text { is r.e. }
$$

To show that $A \cdot B$ is not r.e. we prove that (3) leads to a contradiction ( ${ }^{8}$ ). Assume that (3) holds. As a preliminary we show that for every set $\omega_{n}$ such that $n \in \omega_{n}$ we can decide whether $(n)=\omega_{n}$ or $(n) \subset_{+} \omega_{n}$. For suppose $n \in \omega_{n}$; since $\omega_{n} \neq 0$ we can find a recursive function $t(x)$ ranging over $\omega_{n}$ (e.g., $t(x)$ $=q_{p(n)}(x)$, see Proposition 1.4). If we generate the sequence $t(0), c(0), t(1)$, $c(1), \cdots$ we will after a finite number of steps either find a $y$ such that $t(y)$ $\neq n$ or a $y$ such that $c(y)=n$; in the former case $(n) \subset_{+} \omega_{n}$ and in the latter case $n \in \gamma$, i.e., $(n)=\omega_{n}$. Using this effective procedure we now describe an effective procedure which enables us to decide for any given r.e. set whether it is empty. Let $x$ be any number. We can find a recursive function $g(n)$ such that $\omega_{o(n)}=\omega_{x}+(n)$; by the Corollary of Proposition 1.3 we can find numbers $p$ and $q$ such that

$$
\omega_{p}=\omega_{x}+(p), \quad \omega_{q}=\omega_{x}+(q), \quad p \neq q .
$$

Test each of the statements $(p)=\omega_{p}$ and $(q)=\omega_{q}$. There are three (not mutually exclusive) possibilities:

$$
\begin{aligned}
& (p) C_{+} \omega_{p}=\omega_{x}+(p), \\
& (q) C_{+} \omega_{q}=\omega_{x}+(q), \\
& (p)=\omega_{p} \&(q)=\omega_{q} .
\end{aligned}
$$

If (a) or (b) holds we have $\omega_{x} \neq 0$. It follows from (3) that $\omega_{x} \subset \omega_{p} \cdot \omega_{q}$. Thus, if (c) holds we have $\omega_{x}=o$. The assumption that (3) is valid has now led to a contradiction. For, since $[o]$ is a nontrivial class, there is no effective procedure to test $\omega_{x}=o$.

A r.e. set is semicreative if its complement is semiproductive. Every creative set is of course semicreative, but there are semicreative sets which are not creative $\left({ }^{9}\right)$. A r.e. set is regular if it belongs to $F-E$, but is neither semicreative nor simple.

(8) The following argument is due to J. R. Shoenfield.

$\left.{ }^{9}\right)$ According to an unpublished result of J. R. Shoenfield. Added in proof, July 15, 1958: This result is now published; see Proc. Amer. Math. Soc. vol. 8 (1957) p. 966. 
Proposition 7.1. There exist exactly denumerably many regular sets.

Proof. Let $\zeta \in Z, \eta \in D, \eta \subset \zeta, \mu=\zeta-\eta$. We claim that $\mu$ is regular. First of all, $\mu$ is r.e., because $\mu=\zeta \cdot \eta^{\prime}$. Secondly, $\mu$ is not recursive, for if it were, $\zeta=\mu+\eta$ would also be recursive. Thus, $\mu \in F-E$. Note that $\mu^{\prime}=\zeta^{\prime}+\eta$, where $\zeta^{\prime}$ and $\eta$ are disjoint, $\zeta^{\prime}$ immune and $\eta \in D$. Hence $\mu^{\prime}$ is not immune, i.e., $\mu$ is not simple. If $\mu^{\prime}$ were semiproductive, $\eta$ could be effectively extended to a r.e. set $\eta_{1}$ such that $\eta C_{+} \eta_{1} \subset \eta+\zeta^{\prime}$. Iterating this effective procedure we would obtain a r.e. sequence $\left\{\eta_{n}\right\}$ of infinite r.e. sets such that

$$
\eta C_{+} \eta_{1} C_{+} \eta_{2} C_{+} \cdots \subset_{\eta}+\zeta^{\prime}
$$

Let $\beta$ denote the sum of all sets occurring in $\left\{\eta_{n}\right\}$. Then $\beta-\eta$ is an infinite r.e. subset of the immune set $\zeta^{\prime}$ which is impossible. Hence $\mu^{\prime}$ is not semiproductive and $\mu$ is regular. Every recursive permutation which interchanges the element $m$ of $\mu$ with an element $x$ of $\mu^{\prime}$, but leaves all other elements fixed, maps $\mu$ onto a regular set $\mu_{x}$ different from $\mu$. Since (keeping $m$ fixed) different choices of $x$ yield different sets $\mu_{x}$ it readily follows that there are exactly denumerably many regular sets.

Definition. The class $A$ is immune if it is infinite, but has no infinite r.e. subclass.

We now classify the r.e. classes in terms of the nature of their complements in $F$ in the same way as this is done for r.e. sets.

Definition. Let $A$ be a r.e. class. Then $A$ is semirecursive if $F-A$ is r.e., creative if $F-A$ is productive, semicreative if $F-A$ is semiproductive, simple if $F-A$ is immune and regular if $F-A$ is neither r.e. nor semiproductive nor immune.

Every creative class is semicreative. The converse is false. For if $\alpha$ is a semicreative, but not creative set, the class $F-\left\{(n) \mid n \in \alpha^{\prime}\right\}$ is semicreative, but not creative. Every r.e. class belongs to exactly one of the four categories: semirecursive, semicreative, simple and regular. Our first task is to show that each of these categories is nonempty. We shall see that this is already the case if we restrict ourselves to c.r.e. classes.

Notation. If $\alpha \in F$, Nodi $\alpha=\{\sigma \mid \sigma \in F \& \alpha \cdot \sigma \neq 0\}$.

TheORem 7.2. Among the c.r.e. classes there are denumerably many in each of the four categories: semirecursive, creative, simple and regular.

Proof. Let $\alpha \in F$. Clearly,

$$
\text { Nodi } \alpha=\sum_{x \in \alpha} L(x), \quad F-\text { Nodi } \alpha=K\left(\alpha^{\prime}\right) \text {. }
$$

Thus Nodi is $\alpha$ c.r.e. and its classification can be studied by investigating $K\left(\alpha^{\prime}\right)$. It is readily verified that

(1) $\alpha^{\prime}$ r.e. $\leftrightarrow K\left(\alpha^{\prime}\right)$ r.e.,

(2) $\alpha^{\prime}$ immune $\leftrightarrow\left(\alpha^{\prime}\right)$ immune. 
We know by Theorem 6.3 that Thus,

(3) $\alpha^{\prime}$ semiproductive $\leftrightarrow K\left(\alpha^{\prime}\right)$ semiproductive $\leftrightarrow K\left(\alpha^{\prime}\right)$ productive.

(4) $\alpha$ recursive $\leftrightarrow$ Nodi $\alpha$ semirecursive,

(5) $\alpha$ simple $\leftrightarrow$ Nodi $\alpha$ simple,

(6) $\alpha$ semicreative $\leftrightarrow$ Nodi $\alpha$ semicreative $\leftrightarrow$ Nodi $\alpha$ creative.

Combining (4), (5) and (6) we get

$\alpha$ regular $\leftrightarrow$ Nodi $\alpha$ regular.

Different choices of $\alpha$ yield different c.r.e. classes Nodi $\alpha$. Since there are denumerably many recursive sets, there are at least denumerably many semirecursive c.r.e. classes, hence exactly denumerably many such classes. For each of the other three categories the denumerability of the collection of all c.r.e. classes in that category is established similarly.

Among the c.r.e. classes those which are semirecursive are characterized by Rice in [17]. He proved that the c.r.e. class $S$ has a r.e. complement in $F$ if and only if $S=O$ or $S$ can be expressed as $\sum_{0}^{\infty} T\left(\rho_{f(n)}\right)$ for some recursive function $f(n)$ with a recursive range.

While the most obvious examples of semirecursive classes are perhaps those which are c.r.e. or have a c.r.e. complement, there are many other semirecursive classes.

TheOREM 7.3. Let $\alpha \in E$. Then $[\alpha]$ is semirecursive. Under the additional assumption $\alpha \neq 0$, neither $[\alpha]$ nor $F-[\alpha]$ is c.r.e.

Proof. The second part is readily verified by the reader; we only prove the first part. Assume $\alpha \in E$. The class $[\alpha]$ is r.e. and it suffices to show that $F-[\alpha]$ is r.e.

$$
\begin{aligned}
& F-[\alpha]=B+C, \text { where } \\
& B=\{\sigma \mid \sigma \in F \& \alpha \Phi \sigma\} \text { and } C=\left\{\sigma \mid \sigma \in F \& \sigma \alpha^{\prime} \neq o\right\} .
\end{aligned}
$$

Since $C$ is r.e. (even c.r.e.), it suffices to prove that $B$ is r.e. The class

$$
B_{n}=\{\sigma \mid \sigma \in F \& n \notin \sigma\}
$$

is r.e. for every $n$, and $B=\sum_{n \in \alpha} B_{n}$. Thus $B$ is r.e.

Since every finite set is recursive one might be tempted to conjecture that every finite class is semirecursive; we do not know, however, whether this is the case $\left({ }^{10}\right)$.

While we have shown that semirecursive classes exist the examples of

(10) Added in proof, July 15,1958 . It is, by a theorem of Friedberg (to be published in the Journal of Symbolic Logic) to the effect that there is a partial recursive function $f_{i}(x)$ such that every r.e. set appears exactly once in the sequence $\left\{\rho f_{i}\right\}$. From this it obviously follows that every class with a finite complement is r.e. and every finite class is semirecursive. 
such classes so far discussed are unsatisfactory in the following respect: whether $S=$ Nodi $\alpha$ for $\alpha \in E$ or $S=[\alpha]$ for $\alpha \in E$, in both cases $S$ is defined in terms of a recursive set. A similar remark can be made about the examples of r.e. classes in the other three categories given in the proof of Theorem 7.2. For if such classes exist at all, it is hardly surprising that some of them can be defined in terms of r.e. sets of similar type. In the following two theorems this situation will be remedied as far as semirecursive and creative classes are concerned.

THEOREM 7.4. If $\alpha \in F-Q$ the class $L(\alpha)$ is semirecursive, but neither $L(\alpha)$ nor $F-L(\alpha)$ is c.r.e.

Proof. $L(\alpha)$ contains the infinite r.e. set $\alpha$, but none of its finite subsets; $F-L(\alpha)$ contains all finite sets, but not $\epsilon$. Thus, neither $L(\alpha)$ nor $F-L(\alpha)$ is c.r.e., because neither is a $\Sigma T$-class. $L(\alpha)$ is r.e. by Proposition 1.6(c). To complete the proof we show that $F-L(\alpha)$ is r.e. Let $a(n)$ be a one-to-one recursive function ranging over $\alpha$ and $p(x, y)$ a recursive function for which $\omega_{p(x, y)}=\omega_{x}-(a(y))$. We define

$$
b(x)=p(k(x), l(x)), \beta=\rho b .
$$

The function $b(x)$ is recursive and the set $\beta$ is r.e. We claim that $F-L(\alpha)$ $=\left\{\omega_{n} \mid n \in \beta\right\}$. Clearly $a l(x) \notin \omega_{b(x)}$, hence $\omega_{n} \in F-L(\alpha)$, for every $n \in \beta$. Suppose $\omega_{t} \in F-L(\alpha)$; let $a(u) \in \alpha-\omega_{t}$. Then

$$
\omega_{b j(t, u)}=\omega_{p(t, u)}=\omega_{t}-(a(u))=\omega_{t},
$$

and $\beta$ contains an index of $\omega_{t}$, namely $b j(t, u)$.

Let us consider the special case of the last theorem obtained by restricting $\alpha$ to $E-Q$. Then $L(\alpha)$ is semirecursive, while neither $L(\alpha)$ nor $F-L(\alpha)$ is c.r.e. On the other hand, $\alpha \in E-Q$ not only implies that $K(\alpha)$ is semirecursive, but also that $F-K(\alpha)$ is c.r.e. (observe the absence of duality).

THEOREM 7.5. The following classes are creative: $Q, E, F-(Z+P)$ and $F-Z$.

Proof. By Theorem 6.4 the class $F-Q$ is productive and has a productive center included in $H$. Since $H C F-E \subset F-Q$ we infer by Proposition 6.5 that $F-E$ is productive. The r.e. classes $Q$ and $E$ are therefore creative. By the Corollary of Theorem 6.5 the classes $Z$ and $Z+P$ are productive. Moreover, $F-Z=(F-(Z+P))+P$, where $P$ is r.e. To complete the proof it is therefore enough to show that $F-(Z+P)$ is r.e. Let ' $\Phi(n, x)$ ' denote the condition: $q_{n}(0), \cdots, q_{n}(x)$ are defined and different. Then $\Phi$ is a r.e. condition and there is a recursive function $t(n)$ such that

$$
y \in \omega_{t(n)} \leftrightarrow(\exists x)(\exists z)\left[y=q_{n}(x) \& \Phi(n, x) \& z<x \& q_{n}(z)>q_{n}(x)\right] .
$$


Clearly, $\omega_{t(n)} \subset \omega_{n}$ for all $n$. We distinguish two cases.

CASE 1 . Either $q_{n}(x)$ is not recursive, or $q_{n}(x)$ is recursive, but not one-toone. Then $\omega_{t(n)} \in Q \subset F-(Z+P)$.

CASE 2. $q_{n}(x)$ is recursive and one-to-one. In this case $\omega_{t(n)}$ consists of all elements in the enumeration $\left\{q_{n}(x)\right\}$ of $\omega_{n}$ which are preceded by at least one greater element. Put

$$
\begin{aligned}
\omega_{n} & =\alpha=\left(a_{0}, a_{1}, \cdots\right), \text { where } a_{x}=q_{n}(x), \\
b_{0} & =a_{0}, \\
b_{n+1} & =\text { the first element in }\left\{a_{x}\right\} \text { which exceeds } b_{n} .
\end{aligned}
$$

Then $\omega_{n}=\omega_{t(n)}+\rho b_{n}$, where $\rho b_{n}$ is an infinite recursive set disjoint from $\omega_{t(n)}$. Thus $\omega_{t(n)} \in F-(Z+P)$. Let $T=\left[\omega_{t(0)}, \omega_{t(1)}, \cdots\right]$. Having verified that $T$ is included in $F-(Z+P)$ we proceed to prove

$$
\gamma \in F-(Z+P) \rightarrow \gamma \in T .
$$

Assume the hypothesis. We distinguish three cases.

CASE 1. $\gamma=0$. Note that $\omega_{t(0)}=o$, because $q_{0}(0)$ is undefined. Hence $\gamma \in T$. CASE 2. $\gamma \neq 0$ and $\gamma \in Q$, say

$$
\gamma=\left(c_{1}, \cdots, c_{p}\right), \text { where } c_{1}<c_{2}<\cdots<c_{p} .
$$

Let $k$ be any fixed number greater than $c_{p}$. There obviously exists a recursive function $q_{i}(x)$ such that

$$
\begin{aligned}
& q_{i}(0)=k, \\
& q_{i}(1)=c_{1}, q_{i}(2)=c_{2}, \cdots, q_{i}(p)=c_{p}, \\
& q_{i}(x)=c_{p} \text { for } x>p .
\end{aligned}
$$

It follows that $\gamma=\omega_{\ell(i)} \in T$.

CASE 3. $\gamma \notin Q$. Since $\gamma \in F-(Z+P)$ the set $\gamma^{\prime}$ has an infinite recursive subset, say $\delta$. Let $c(n)$ be a one-to-one recursive function ranging over $\gamma$. Put

$$
\begin{aligned}
e(0) & =(\mu y)[y \in \delta \& y>c(0)], \\
e(n+1) & =(\mu y)[y \in \delta \& y>\max (e(n), c(n+1))] .
\end{aligned}
$$

Then $e(n)$ is a strictly increasing recursive function such that (a) $e(i)$ and $c(i)$ are less than $e(n+1)$ for $0 \leqq i \leqq n$, (b) $e(n)>c(n)$ for all $n$. Let us now consider the sequence

$$
e(0), c(0), e(1), c(1), \cdots .
$$

$\mathrm{By}$ (a) the element $e(x)$ is greater than all its predecessors in (I) and by (b) the element $c(x)$ is less than its immediate predecessor in (I). The function $h(n)$ defined by $h(2 n)=e(n), h(2 n+1)=c(n)$ is one-to-one and recursive. If $r$ is a number such that $h(x)=q_{r}(x)$ we have $\gamma=\omega_{\iota(r)} \in T$. 
Proposition 7.2. Let $A$ be a creative class, $B$ a r.e. class and $B \subset F-A$. Then $A+B$ is creative.

Proof. Use the first part of Proposition 6.7 and the fact that the sum of two r.e. classes is r.e.

Once we know that certain r.e. classes are creative one can establish the creativity of many other r.e. classes using Proposition 7.2. For example, $P+Q$ is creative, because $Q$ is creative and $P$ is a r.e. subclass of $F-Q$.

\section{REFERENCES}

1. M. Davis, Computability and unsolvability, McGraw-Hill, New York, 1958.

2. J. C. E. Dekker, The constructivity of maximal dual ideals in certain Boolean algebras, Pacific J. of Math. vol. 3 (1953) pp. 73-101.

3. - Two notes on recursively enumerable sets, Proc. Amer. Math. Soc. vol. 4 (1953) pp. $495-501$.

4. - Productive sets, Trans. Amer. Math. Soc. vol. 78 (1955) pp. 129-149.

5. J. C. E. Dekker and J. Myhill, Recursion theory, to be published by the North Holland Pub. Co., Amsterdam.

6. R. M. Friedberg, The solution of Post's problem by the construction of two recursively enumerable sets of incomparable degree of unsolvability, Bull. Amer. Math. Soc. Abstract 62-3-362.

7. S. C. Kleene, Introduction to metamathematics, New York, Van Nostrand, Amsterdam, North Holland Pub. Co., and Groningen, Noordhoff, 1952, x+550 pp.

8. S. C. Kleene and E. L. Post, The upper semi-lattice of degrees of recursive unsolvability, Ann. of Math. vol. 59 (1954) pp. 379-407.

9. R. McNaughton, On the effective distinguishability of classes of theories, abstract, J. Symbolic Logic vol. 19 (1954) p. 157. 112.

10. A. Mostowski, On definable sets of positive integers, Fund. Math. vol. 34 (1947) pp. 81-

11. On a set of integers not definable by means of one quantifier predicates, Ann. Soc. Polon. Math. vol. 21 (1948) pp. 114-119.

12. A. A. Muchnik, Negative answer to the problem of reducibility of the theory of algorithms, C. R. (Doklady) Acad. Sci. URSS vol. 108 (1956) pp. 194-197.

13. J. R. Myhill, Creative sets, Zeitschrift fur Mathematische Logik und Grundlagen der Mathematik vol. 1 (1955) pp. 97-108.

14. J. R. Myhill and J. C. Shepherdson, Effective operations on partial recursive functions, ibid. vol. 1 (1955) pp. 310-317.

15. E. L. Post, Recursively enumerable sets of positive integers and their decision problems, Bull. Amer. Math. Soc. vol. 50 (1944) pp. 284-316.

16. H. G. Rice, Classes of recursively enumerable sets and their decision problems, Trans. Amer. Soc. vol. 74 (1953) pp. 358-366.

17. - On completely recursively enumerable classes and their key arrays, J. Symbolic Logic vol. 21 (1956) pp. 304-308. 299.

18. N. Shapiro, Degrees of computability, Trans. Amer. Math. Soc. vol. 82 (1956) pp. 281-

University of Chicago,

Chicago, Ill.

Institute for Advanced Study,

Princeton, N. J.

University of California,

Berkeley, Calif. 